\title{
Ototoxicity from Combined Cisplatin and Radiation Treatment: An In Vitro Study
}

\author{
Wong-Kein Low, ${ }^{1}$ Sylvia W. W. Kong, ${ }^{2}$ and Michelle G. K. Tan ${ }^{2}$ \\ ${ }^{1}$ Department of Otolaryngology, Singapore General Hospital, Singapore 169608 \\ ${ }^{2}$ Department of Clinical Research, Singapore General Hospital, Singapore 169608 \\ Correspondence should be addressed to Wong-Kein Low, low.wong.kein@sgh.com.sg
}

Received 10 June 2010; Revised 29 July 2010; Accepted 10 August 2010

Academic Editor: Leonard P. Rybak

Copyright (C) 2010 Wong-Kein Low et al. This is an open access article distributed under the Creative Commons Attribution License, which permits unrestricted use, distribution, and reproduction in any medium, provided the original work is properly cited.

\begin{abstract}
Objective. Combined cisplatin (CDDP) and radiotherapy is increasingly being used to treat advanced head and neck cancers. As both CDDP and radiation can cause hearing loss, it is important to have a better understanding of the cellular and molecular ototoxic mechanisms involved in combined therapy. Procedure. The effects of CDDP, radiation, and combined CDDP-radiation on the OC-k3 cochlear cell line were studied using MTS assay, flow cytometry, Western blotting, and microarray analysis. Results. Compared to using CDDP or radiation alone, its combined use resulted in enhanced apoptotic cell death and apoptotic-related gene expression, including that of FAS. Phosphorylation of p53 at Ser15 (a marker for p53 pathway activation in response to DNA damage) was observed after treatment with either CDDP or radiation. However, posttreatment activation of p53 occurred earlier in radiation than in CDDP which corresponded to the timings of MDM2 and TP53INP1 expression. Conclusion. Enhanced apoptotic-related gene expressions leading to increased apoptotic cell deaths could explain the synergistic ototoxicity seen clinically in combined CDDP-radiation therapy. CDDP and radiation led to differential temporal activation of p53 which suggests that their activation is the result of different upstream processes. These have implications in future antiapoptotic treatments for ototoxicity.
\end{abstract}

\section{Introduction}

Combined chemoradiotherapy is increasingly being used to treat advanced head and neck cancers. During radiotherapy, the ear structures are often included in the radiation fields and it is generally accepted that radiation-induced sensorineural hearing loss can result. Cisplatin (CDDP), widely used as an effective antineoplastic drug for these cancers, is also known to cause ototoxicity. In a randomized blinded study, it was demonstrated that patients who had received radiotherapy and concurrent/adjuvant chemotherapy using CDDP experienced greater sensorineural hearing loss compared with patients treated with radiotherapy alone [1]. This was especially so in the high-frequency sounds of the speech range, resulting in significant hearing disability.

In recent years, immortalized cell lines derived from the mouse organ of Corti had been developed and characterized [2]. For example, the OC-k3 cell line was derived from the organ of Corti of the transgenic mouse. It encoded the large
T antigen of the SV40 (simian virus 40), a thermolabile viral protein which drove the cells to proliferate indefinitely at $33^{\circ} \mathrm{C}$ and in the presence of gamma interferon [3]. This cell line expressed the neuro-epithelial precursor cell marker nestin and the inner ear cell marker OCP2, but did not exhibit markers for glial or neuronal cells. In addition, OCk3 cells expressed specific auditory sensory cell markers (myosin VIIa and the acetylcholine receptor alpha-9) and the supporting cell marker connexin 26 . This and other similar cell lines had been regarded as good models to study the mechanisms of cell fate in the organ of Corti of the cochlea [4].

P53 had been found to play an important role in apoptotic cell death associated with ototoxicity. In a CDDPinduced apoptosis experiment using cochlear organotypic cultures prepared from rats at postnatal days 3-4, significant upregulation of phospho-p53 serine 15 expression was found and apoptosis was suppressed by pifithrin- $\alpha$, a p53 inhibitor [5]. Other studies have shown that the deletion of the p53 
gene protects sensory hair cells from CDDP-induced cell death, caspase- 2 activation, and cytochrome $\mathrm{c}$ translocation [6]. In radiation-induced ototoxicity, it was found that p53 together with reactive oxidative species (ROS) played an important role in cochlear cell apoptosis [7].

In the combined use of CDDP and radiation, the cellular and molecular mechanisms leading to ototoxicity had not been studied. It is important to have a better understanding of these mechanisms as effective preventive strategies directed at the relevant pathways can potentially be developed. The present study found that although p53 played a role in both CDDP and radiation-induced cochlear cell apoptosis, p53 was activated at different time points after each treatment which corresponded to the time MDM2 and TP53INP1 were expressed. Additional apoptotic-related genes that were not expressed when CDDP or radiation was used alone were expressed when used in combination. This included FAS, an important element involved in the extrinsic apoptotic pathway.

\section{Materials and Methods}

2.1. Cell Culture. The immortalized OC-k 3 cell line derived from the organ of Corti of the transgenic mice (Immortamouse $\mathrm{H}-2 \mathrm{~Kb}$-tsA58, Charles Rivers Laboratories, Wilmington, MA) was used. The cell line was cultured in high-glucose Dulbecco's Eagle's medium (DMEM, Gibco, Grand Island, NY) supplemented with $10 \%$ fetal bovine serum (FBS, Gibco, Grand Island, NY), 1\% penicillin-streptomycin (P/S, Gibco, Grand Island, NY), and $50 \mathrm{U} / \mathrm{ml}$ gamma-interferon (mouse recombinant, Sigma-Aldrich, St. Louis, MO) and maintained at $33^{\circ} \mathrm{C}$ with $10 \% \mathrm{CO}_{2}$. To study the impact of chemoradiation treatment, OC-k3 cells were exposed to 5 Gy of gamma irradiation alone, $0.5 \mu \mathrm{g} / \mathrm{ml}$ of cisplatin alone, or $5 \mathrm{~Gy}$ of gamma irradiation in the presence of $0.5 \mu \mathrm{g} / \mathrm{ml}$ cisplatin (Pfizer, Bentley, WA).

2.2. Cell Viability Assay. The OC-k3 cells were seeded in 96-well plates at densities of $5 \times 10^{3}$ cells/well in $200 \mu \mathrm{l}$ complete medium after being exposed to chemo-irradiation treatment. Cell viability was determined using CellTiter 96 Aqueous One Solution Cell Proliferation Assay (Promega Corp., Madison, WI) containing tetrazolium compound 3-[4,5-dimethylthiazol-2-yl]-5-(3-carboxymethoxyphenyl)2-(4-sulfophenyl)-2H-tetrazolium (MTS) at $3 \mathrm{~h}, 24 \mathrm{~h}, 48 \mathrm{~h}$, and $72 \mathrm{~h}$ after chemo-irradiation. This test was based on the bioreduction of MTS compound into a soluble and colored formazan product by NADPH or NADH, which is produced by dehydrogenase enzymes in metabolically active cells. Twenty microliters of MTS were added to each well, incubated at $33^{\circ} \mathrm{C}$ for $3 \mathrm{~h}$, and then the absorbance was recorded at $490 \mathrm{~nm}$ with a microplate spectrophotometer (Benchmark Plus, Bio-Rad Laboratories, Hercules, CA).

2.3. Cell Death Analysis. The cells were collected at each time point post CDDP-radiation treatment, fixed in $75 \%$ ethanol and stored at $4^{\circ} \mathrm{C}$. Upon analysis, the cells were washed with PBS and incubated with $100 \mu \mathrm{g} / \mathrm{ml}$ propidium iodide
(PI) containing $0.1 \%$ Triton X-100 and $500 \mu \mathrm{g} / \mathrm{ml}$ RNase $\mathrm{A}$ in $50 \mu \mathrm{l}$ PBS for 30 mins in darkness at $4^{\circ} \mathrm{C}$. The DNA contents of cells were analyzed using the flow cytometer CyAnTM ADP Analyser (Beckman Coulter, Fullerton, CA). The magnitudes of the sub-G1 fractions were determined using the Summit 4.3 software (Beckman Coulter, Fullerton, CA). DNA fragmentation resulting from apoptotic cell death would manifest in the sub-G1 fraction.

2.4. Western Blot Analysis. Protein extraction was done by incubating the cells at $4^{\circ} \mathrm{C}$ for 30 minutes in lysis buffer containing $150 \mathrm{mM} \mathrm{NaCl}, 10 \mathrm{mM}$ Tris- $\mathrm{HCl} \mathrm{pH} \mathrm{7.4,} 2$ mM EDTA, $0.5 \mathrm{mM}$ EGTA, $1 \mathrm{mM}$ sodium orthovanadate, $0.1 \%$ sodium deoxycholate, $0.5 \%$ NP-40, and $1 \%$ Triton X-100 supplemented with $1 \mathrm{x}$ complete protease inhibitor mixture (Roche, Basel, Switzerland). Equal amounts of protein samples were denatured separated by 10\% SDS-PAGE and transferred onto nitrocellulose membrane by iBlot dry blotting system (Invitrogen, Carlsbad, CA). The membrane was blocked with $5 \%$ nonfat milk in PBS with $0.1 \%$ Tween-20 (PBST) for $1 \mathrm{~h}$, followed by an overnight incubation of primary antibodies in $5 \%$ BSA/PBST at $4^{\circ} \mathrm{C}$. Primary antibodies included antip53 pAb (NCL-p53-CM5p, Novocastra), anti-phospho-p53 (ser-15) pAb, anti-phospho-c-jun (ser-73) pAb, anti-c-jun (60A8) mAb (Cell Signaling Technology, Inc.), and anti-betaactin $\mathrm{mAb}$ (Sigma-Aldrich, St. Louise, MO). After washing the membrane extensively, incubation with horseradish peroxidase-conjugated antirabbit or antimouse secondary antibody (Cell Signaling Technology, Inc.) was done for $1 \mathrm{~h}$ at room temperature. After washing, the membrane was incubated in Immobilon Western chemiluminescent HRP substrate (Millipore, Billerica, MA), and the chemiluminescence signals were detected using UVIchemi (UVItec, Cambridge, UK), a dedicated chemiluminescence documentation system. For reprobing with a new primary antibody, the membrane was stripped in Re-Blot plus strong solution Western blot stripping buffer (Chemicon, Temecula, CA) at room temperature for 30 minutes and rinsed 3 times with PBST for 10 minutes each time.

2.5. Microarray Analysis. The global changes of gene expression were analyzed at $3 \mathrm{~h}, 24 \mathrm{~h}$, and $72 \mathrm{~h}$ after chemoirradiation, on the GeneChip Mouse Genome 430A 2.0 Array (Affymetrix, Santa Clara, CA). Biological duplicates of experiments were performed. Briefly, RNA was extracted from cells using TRIzol reagent (Invitrogen, Carlsbad, CA) followed by generation of double-stranded cDNA. These were used as templates for synthesis of biotin-labeled cRNA, using the GeneChip IVT labeling kit in accordance with the manufacturer's instructions. The biotinylated cRNA was purified using RNeasy Mini kit (Qiagen, Hilden, Germany) and fragmented before reconstitution in a hybridization cocktail mixture containing eukaryotic hybridization control. The hybridization was performed at $45^{\circ} \mathrm{C}$ for $16 \mathrm{~h}$ in a rotisserie oven set at $60 \mathrm{rpm}$. Upon completion, the arrays were then loaded onto an Affymetrix Fluidic station, washed according to the standard Affymetrix EukGEWS2v5 protocol and stained with streptavidin-phycoerythrin 


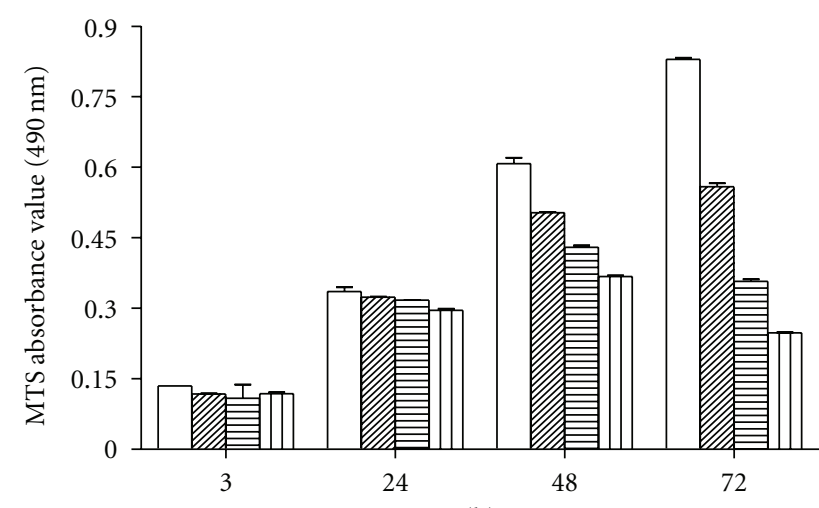

(h)
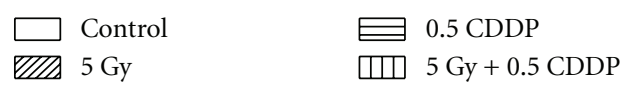

Figure 1: Cell viability analysis by MTS assay at different time points $(3 \mathrm{~h}, 24 \mathrm{~h}, 48 \mathrm{~h}$, and $72 \mathrm{~h}$ ) after treatment with $5 \mathrm{~Gy}$ gamma radiation and $0.5 \mu \mathrm{g} / \mathrm{ml}$ cisplatin (CDDP). After co-treatment with radiation and CDDP, cell viability was significantly reduced at $72 \mathrm{~h}$. The data shown are the most representative of 3 separate experiments.

(SAPE) solution. After washing and staining, the arrays were scanned with the Gene Array scanner (Affymetrix, Santa Clara, CA). Hybridization intensity data detected by the scanner were automatically acquired and processed by the Affymetrix GeneChip Operating Software (GCOS, Affymetrix, Santa Clara, CA). The average intensity for all the genes was normalized to 100 . The statistical algorithms implemented in GCOS software were used for analysis. In a comparison expression analysis, each probe pair on the experimental array was compared to the corresponding probe pair on the baseline array (control). This generated an associated "change" (increased, no change, or decreased) to determine the relative expression of transcripts. To have an overview of gene expression profiles, probe sets showing chemoradiation-induced increased or decreased expressions in both duplicated experiments were retrieved. The differentially expressed genes of chemoradiation treatment were submitted for biological functional analysis using Ingenuity Pathway Analysis (IPA) tools (Ingenuity Systems, http://www.ingenuity.com).

\section{Results}

3.1. Combined CDDP-Radiation Treatment Reduced Cell Viability More than CDDP or Radiation Treatment Alone. Cell viability analysis by MTS assay at different time points revealed that although CPPD and radiation each exerted a negative effect on cell viability, treatment when combined appeared to have a greater effect. These effects were observed at $48 \mathrm{hrs}$ after treatment and became even more marked at 72 hrs after treatment (Figure 1).

3.2. Apoptosis Occurred Predominantly at $72 \mathrm{~h}$ after Combined CDDP-Radiation Treatment. At $72 \mathrm{hrs}$ after treatment,

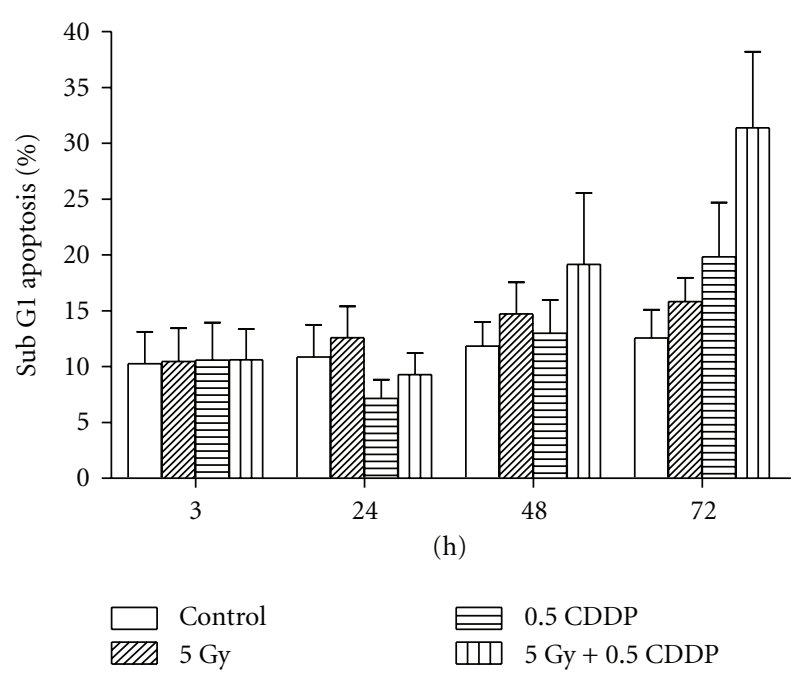

FIgURE 2: Flow cytometric subG1 phase as determined by PI staining at different time points $(3 \mathrm{~h}, 24 \mathrm{~h}, 48 \mathrm{~h}$ and $72 \mathrm{~h}$ ) after exposure to $5 \mathrm{~Gy}$ of gamma radiation and $0.5 \mu \mathrm{g} / \mathrm{ml}$ of cisplatin (CDDP). Co-treatment with radiation and CDDP resulted in a significant increase in subG1 phase at $72 \mathrm{~h}$. The data shown are the mean $+\mathrm{SD}$ of 4 independent experiments.

combined CDDP-radiation led to a greater increase in the sub-G1 phase as compared to using CDDP and radiation alone (Figure 2). As pointed out previously, DNA fragmentation resulting from apoptotic cell death manifests in the sub-G1 fraction.

3.3. Apoptosis-Related Gene Expressions were Enhanced by Combined CDDP-Radiation Treatment. On analyzing the results of molecular and cellular functions under the biological functions of IPA, it was found that among the 3925 probe set IDs which were differentially expressed in at least one treatment, 942 represented 623 unique genes associated with apoptosis (see Table 1). Their distribution at each time point for the different treatment regimes is summarized in Venn diagrams (Figure 3). A subset focusing on the genes, which had a direct upstream or downstream relationship with p53, is shown in Table 2. Combined CDDP-radiation treatment resulted in an increase in the number of gene expressions which was more than merely a summation of the number of expressions resulting from individual treatments (Figure 3, Table 2). At 72 hrs after treatment, 40 out of the 163 genes listed $(24.5 \%)$ were expressed in combined CDDP-radiation treatment, but not when CDDP or radiation was used alone (Table 2). Among these 40 genes was FAS, an important element of the extrinsic apoptotic pathway.

3.4. Differential Temporal Activation of p53 Occurred with CDDP and Radiation Treatment. It was observed that Posttreatment activation of $\mathrm{p} 53$ occurred earlier in radiation than in CDDP (Figure 4). In response to DNA damage, activation of the p53 pathway normally occurs with the phosphorylation of ser-15 in p53. The present study showed radiation-induced phosphorylation of p53 occurred at $3 \mathrm{hrs}$ 
TABLE 1: Differentially expressed apoptosis-related genes in each treatment group [irradiation (Gy), cisplatin (CDDP), or combination of both $($ Gy + CDDP) ] when compared to nontreated control cells at $3 \mathrm{~h}, 24 \mathrm{~h}$, and $72 \mathrm{~h}$ after treatment

\begin{tabular}{|c|c|c|c|c|c|c|c|c|c|c|}
\hline & & & $3 \mathrm{~h}$ & & & $24 \mathrm{~h}$ & & & $72 \mathrm{~h}$ & \\
\hline Symbol & Probe set ID & Gy & CDDP & $\begin{array}{l}\text { Gy }+ \\
\text { CDDP }\end{array}$ & Gy & CDDP & $\begin{array}{l}\text { Gy }+ \\
\text { CDDP }\end{array}$ & Gy & CDDP & $\begin{array}{l}\text { Gy }+ \\
\text { CDDP }\end{array}$ \\
\hline AAK1 & $\begin{array}{l}\text { 1420025_s_at, } \\
\text { 1434935_at }\end{array}$ & & & & & & & & I & I \\
\hline AARS & 1423685_at & & & & & & & & & $\mathrm{D}$ \\
\hline $\mathrm{ABCB} 1 \mathrm{~B}$ & 1418872_at & & & & & & & & I & I \\
\hline ABCC1 & 1452233_at & & & & & & & & & I \\
\hline ABCG2 & 1422906_at & & & & & & & & & I \\
\hline ABL2 & 1455495_at & & & & & & & & & I \\
\hline ACSL4 & 1451828_a_at & & & & & & & & & I \\
\hline ACTN4 & 1423449_a_at & & & & & & & & & I \\
\hline ACVR1 & 1448460_at & & & & & $\mathrm{D}$ & & & & \\
\hline ADAMTSL4 & 1451932_a_at & & & & & & & & & I \\
\hline $\mathrm{ADM}$ & $\begin{array}{l}\text { 1416077_at, } \\
\text { 1447839_x_at }\end{array}$ & & & & & $\mathrm{D}$ & $\mathrm{D}$ & I & & I \\
\hline AES & 1420619_a_at & & & & & & $\mathrm{D}$ & & & \\
\hline AFP & 1416645_a_at & & & & & & & & & I \\
\hline AHR & 1422631_at & & & & & & & & & I \\
\hline AIMP1 & 1416486_at & & & & & & & & & $\mathrm{D}$ \\
\hline AKAP12 & 1419706_a_at & & & & & & & & I & I \\
\hline AKT1S1 & $\begin{array}{l}\text { 1428158_at, } \\
\text { 1452684_at }\end{array}$ & & & & & & & & & $\mathrm{D}$ \\
\hline ALDH1A1 & 1416468_at & & & & & & & & I & I \\
\hline ALDH1A2 & 1422789_at & & & & & & & & & $\mathrm{D}$ \\
\hline ALDOA & $\begin{array}{l}\text { 1416921_x_at, } \\
\text { 1433604_x_at, } \\
\text { 1434799_x_at, } \\
\text { 1439375_x_at }\end{array}$ & & & & & $\mathrm{D}$ & $\mathrm{D}$ & I & I & I \\
\hline ANKRD1 & $\begin{array}{l}\text { 1420991_at, } \\
\text { 1420992_at }\end{array}$ & & & & & & & I & I & I \\
\hline ANLN & $\begin{array}{l}\text { 1433543_at, } \\
\text { 1439648_at }\end{array}$ & & & & & & & & I & I \\
\hline ANP32A & 1421918_at & & & & & $\mathrm{D}$ & & & & \\
\hline ANXA1 & 1448213_at & & & & & & & I & I & I \\
\hline ANXA7 & 1416138_at & & & & & & & & I & I \\
\hline AP2A2 & 1452490_a_at & & & & & & & & & I \\
\hline APBB2 & 1452342_at & & & & & & & & & I \\
\hline APEX1 & $\begin{array}{l}\text { 1416135_at, } \\
\text { 1437715_x_at, } \\
\text { 1456079_x_at }\end{array}$ & & & & & $\mathrm{D}$ & $\mathrm{D}$ & & & \\
\hline APOE & 1432466_a_at & & & & & & & I & I & I \\
\hline APPL1 & 1455159_at & & & & & I & & & & \\
\hline ARNT2 & 1434028_at & & & & & & & & & I \\
\hline ASAH2 & 1450726_at & & & & & & & & I & I \\
\hline ATF3 & 1449363_at & & & $\mathrm{D}$ & & & & $\mathrm{D}$ & & $\mathrm{D}$ \\
\hline ATF5 & 1425927_a_at & & & & & & & $\mathrm{D}$ & & $\mathrm{D}$ \\
\hline ATG12 & 1451746_a_at & & & & & & & & $\mathrm{D}$ & $\mathrm{D}$ \\
\hline ATG5 & 1418235_at & & & I & & & & & & \\
\hline ATM & $\begin{array}{l}\text { 1421205_at, } \\
\text { 1428830_at }\end{array}$ & & & & & & & & I & I \\
\hline
\end{tabular}


Table 1: Continued.

\begin{tabular}{|c|c|c|c|c|c|c|c|c|c|c|}
\hline & & & $3 \mathrm{~h}$ & & & $24 \mathrm{~h}$ & & & $72 \mathrm{~h}$ & \\
\hline Symbol & Probe set ID & Gy & CDDP & $\begin{array}{l}\text { Gy }+ \\
\text { CDDP }\end{array}$ & Gy & CDDP & $\begin{array}{l}\text { Gy }+ \\
\text { CDDP }\end{array}$ & Gy & CDDP & $\begin{array}{l}\text { Gy }+ \\
\text { CDDP }\end{array}$ \\
\hline ATN1 & 1421149_a_at & & & & & & & & I & \\
\hline ATP1A1 & 1451071_a_at & & & & & & $\mathrm{D}$ & & & \\
\hline ATP7A & 1436921_at & & & & & I & & & & \\
\hline ATXN2 & 1460653_at & & & $\mathrm{D}$ & & & & & & \\
\hline AURKA & 1424511_at & & & $\mathrm{D}$ & & & & I & I & I \\
\hline AXL & 1423586_at & & & D & & & & & I & I \\
\hline BAG3 & 1422452_at & & & $\mathrm{D}$ & & & & & & \\
\hline BANF1 & $\begin{array}{l}\text { 1421081_a_at, } \\
\text { 1421082_s_at, } \\
\text { 1421083_x_at }\end{array}$ & & & & & & & & $\mathrm{D}$ & $\mathrm{D}$ \\
\hline BCAR1 & $\begin{array}{l}\text { 1439388_s_at, } \\
\text { 1450622_at }\end{array}$ & & & $\mathrm{D}$ & & & & & I & I \\
\hline BCL3 & 1418133_at & & & & & & & I & & I \\
\hline BCLAF1 & $\begin{array}{l}\text { 1428844_a_at, } \\
\text { 1428845_at, } \\
\text { 1436023_at, } \\
\text { 1438089_a_at }\end{array}$ & & & & I & I & & & & \\
\hline BDNF & 1422168_a_at & & & & & & & & & I \\
\hline BECN1 & $\begin{array}{l}\text { 1455880_s_at, } \\
\text { 1460320_at }\end{array}$ & & & & & & & & I & I \\
\hline BGN & $\begin{array}{l}\text { 1437889_x_at, } \\
\text { 1448323_a_at }\end{array}$ & & & & & $\mathrm{D}$ & $\mathrm{D}$ & & & I \\
\hline BHLHE40 & 1418025_at & & & & & D & $\mathrm{D}$ & & & I \\
\hline BID & $\begin{array}{l}\text { 1417045_at, } \\
\text { 1448560_at }\end{array}$ & & & D & & & & & I & I \\
\hline BIRC3 & 1421392_a_at & & & & & & & & & I \\
\hline BIRC5 & 1424278_a_at & & & & & & & & I & I \\
\hline BLM & 1448953_at & & & & & I & & & & \\
\hline BNIP2 & 1453993_a_at & & & & & & & & & $\mathrm{D}$ \\
\hline BNIP3 & 1422470_at & & & & & & & $\mathrm{D}$ & & \\
\hline BPTF & $\begin{array}{l}\text { 1427310_at, } \\
\text { 1456615_a_at }\end{array}$ & & & & & I & & & & \\
\hline BRAF & 1435434_at & & & & & I & & & & \\
\hline BRCA1 & $\begin{array}{l}\text { 1424629_at, } \\
\text { 1424630_a_at, } \\
\text { 1451417_at }\end{array}$ & & & & & I & & & & \\
\hline BRD2 & $\begin{array}{l}\text { 1423502_at, } \\
\text { 1437210_a_at }\end{array}$ & & & & & $\mathrm{D}$ & $\mathrm{D}$ & & & $\mathrm{D}$ \\
\hline BRE & $\begin{array}{l}\text { 1426312_at, } \\
\text { 1426313_at }\end{array}$ & & & & & $\mathrm{D}$ & $\mathrm{D}$ & & & $\mathrm{D}$ \\
\hline BTG1 & 1426083_a_at & & & & & $\mathrm{D}$ & & & & \\
\hline BTG2 & $\begin{array}{l}\text { 1416250_at, } \\
\text { 1448272_at }\end{array}$ & I & & & & & I & & & \\
\hline BUB1 & 1424046_at & & & & & I & I & & I & I \\
\hline BUB1B & $\begin{array}{l}\text { 1416961_at, } \\
\text { 1447363_s_at }\end{array}$ & & & & & & & I & I & I \\
\hline C11ORF82 & 1429734_at & & & I & & & I & & & I \\
\hline C1QBP & 1455821_x_at & & & & & & & & $\mathrm{D}$ & \\
\hline
\end{tabular}


Table 1: Continued.

\begin{tabular}{|c|c|c|c|c|c|c|c|c|c|c|}
\hline & & & $3 \mathrm{~h}$ & & & $24 \mathrm{~h}$ & & & $72 \mathrm{~h}$ & \\
\hline Symbol & Probe set ID & Gy & CDDP & $\begin{array}{l}\text { Gy }+ \\
\text { CDDP }\end{array}$ & Gy & CDDP & $\begin{array}{l}\text { Gy }+ \\
\text { CDDP }\end{array}$ & Gy & CDDP & $\begin{array}{l}\mathrm{Gy}+ \\
\mathrm{CDDP}\end{array}$ \\
\hline C3 & 1423954_at & & & & & & & I & & I \\
\hline CACNA1A & 1450510_a_at & & & & & & & & I & \\
\hline CACNA1C & 1421297_a_at & & & & & & & & $\mathrm{D}$ & $\mathrm{D}$ \\
\hline CASP12 & 1449297_at & & & & & & & $\mathrm{D}$ & & \\
\hline CASP2 & 1448165_at & & & & & & & $\mathrm{D}$ & & \\
\hline CASP3 & $\begin{array}{l}\text { 1426165_a_at, } \\
\text { 1449839_at }\end{array}$ & & & I & & & & & & \\
\hline CASP6 & 1415995_at & & & I & & & & & & I \\
\hline CASP7 & $\begin{array}{l}\text { 1426062_a_at, } \\
\text { 1448659_at }\end{array}$ & & & & & & & $\mathrm{D}$ & & $\mathrm{D}$ \\
\hline CASP9 & 1426125_a_at & $\mathrm{D}$ & & & & & & & & \\
\hline CAST & $\begin{array}{l}\text { 1426098_a_at, } \\
\text { 1435972_at, } \\
1451413 \_a t\end{array}$ & & & & & I & I & I & I & I \\
\hline CAT & 1416429_a_at & & & & & & & & & I \\
\hline CAV1 & 1449145_a_at & & & & & $\mathrm{D}$ & & I & I & I \\
\hline CBX5 & $\begin{array}{l}\text { 1421933_at, } \\
\text { 1450416_at }\end{array}$ & & & & & & & $\mathrm{D}$ & & $\mathrm{D}$ \\
\hline CCAR1 & $\begin{array}{l}\text { 1436156_at, } \\
\text { 1436157_at }\end{array}$ & & & & & I & I & & & \\
\hline CCL13 & 1420380_at & & & & & & I & I & I & I \\
\hline CCL5 & 1418126_at & & & & I & & I & & I & I \\
\hline CCL9 & $\begin{array}{l}\text { 1417936_at, } \\
\text { 1448898_at }\end{array}$ & & & & & & & & I & I \\
\hline CCNA2 & $\begin{array}{l}\text { 1417910_at, } \\
\text { 1417911_at }\end{array}$ & & & & & & & & I & I \\
\hline CCNB1 & $\begin{array}{l}\text { 1416076_at, } \\
\text { 1419943_s_at, } \\
\text { 1448205_at, } \\
\text { 1449675_at }\end{array}$ & & & $\mathrm{D}$ & & & & I & I & I \\
\hline CCND1 & $\begin{array}{l}\text { 1417419_at, } \\
\text { 1417420_at, } \\
\text { 1448698_at }\end{array}$ & $\mathrm{D}$ & & & & & & I & I & I \\
\hline CCND3 & 1415907_at & & & & & & & & I & I \\
\hline CCNG1 & $\begin{array}{l}\text { 1420827_a_at, } \\
\text { 1450016_at, } \\
\text { 1450017_at }\end{array}$ & I & & I & I & I & I & I & I & I \\
\hline CD14 & 1417268_at & & & & & & & & & I \\
\hline $\mathrm{CD} 24$ & $\begin{array}{l}\text { 1416034_at, } \\
\text { 1437502_x_at, } \\
\text { 1448182_a_at }\end{array}$ & & & $\mathrm{D}$ & & & & & & I \\
\hline CD274 & 1419714_at & & & & & & I & $\mathrm{D}$ & & \\
\hline CD2AP & 1420907_at & & & & & I & & & & \\
\hline $\mathrm{CD} 44$ & $\begin{array}{l}\text { 1423760_at, } \\
\text { 1434376_at, } \\
\text { 1452483_a_at }\end{array}$ & & & & & & & & I & I \\
\hline CD47 & $\begin{array}{l}\text { 1419554_at, } \\
\text { 1428187_at, } \\
\text { 1449507_a_at }\end{array}$ & & & & & & & $\mathrm{D}$ & & $\mathrm{D}$ \\
\hline
\end{tabular}


Table 1: Continued.

\begin{tabular}{|c|c|c|c|c|c|c|c|c|c|c|}
\hline & & & $3 \mathrm{~h}$ & & & $24 \mathrm{~h}$ & & & $72 \mathrm{~h}$ & \\
\hline Symbol & Probe set ID & Gy & CDDP & $\begin{array}{l}\text { Gy }+ \\
\text { CDDP }\end{array}$ & Gy & CDDP & $\begin{array}{l}\text { Gy }+ \\
\text { CDDP }\end{array}$ & Gy & CDDP & $\begin{array}{l}\text { Gy + } \\
\text { CDDP }\end{array}$ \\
\hline CD80 & 1432826_a_at & & & & & & & & & I \\
\hline CD9 & 1416066_at & & & $\mathrm{D}$ & & & & I & I & I \\
\hline CDC20 & $\begin{array}{l}\text { 1416664_at, } \\
\text { 1439377_x_at }\end{array}$ & & & & & & & I & I & I \\
\hline CDC25B & 1421963_a_at & & & & & & & & I & I \\
\hline CDC25C & $\begin{array}{l}\text { 1422252_a_at, } \\
\text { 1456077_x_at }\end{array}$ & & & & & & & & I & I \\
\hline CDC2L2 & 1418841_s_at & & & & & & & & & $\mathrm{D}$ \\
\hline CDC37 & 1416819_at & & & & & & & & I & I \\
\hline CDC42EP3 & $\begin{array}{l}\text { 1422642_at, } \\
\text { 1450700_at }\end{array}$ & & & & & & & I & I & I \\
\hline CDC45L & 1416575_at & & & & & & & $\mathrm{D}$ & & $\mathrm{D}$ \\
\hline CDC6 & 1417019_a_at & & & & & & & & & $\mathrm{D}$ \\
\hline CDCA2 & $\begin{array}{l}\text { 1437251_at, } \\
\text { 1455983_at }\end{array}$ & & & & & & & & I & I \\
\hline $\mathrm{CDH} 2$ & 1418815_at & & & & & & & & & I \\
\hline CDK4 & $\begin{array}{l}\text { 1422439_a_at, } \\
\text { 1422440_at, } \\
\text { 1422441_x_at }\end{array}$ & & & & & & $\mathrm{D}$ & & $\mathrm{D}$ & $\mathrm{D}$ \\
\hline CDK8 & 1460389_at & & & & & & & & & I \\
\hline CDKN1A & $\begin{array}{l}\text { 1421679_a_at, } \\
\text { 1424638_at }\end{array}$ & I & & I & I & I & I & I & I & I \\
\hline CDKN1B & 1434045_at & & & & & & & & $\mathrm{D}$ & $\mathrm{D}$ \\
\hline CDKN2A & 1450140_a_at & & & & & & $\mathrm{D}$ & & & \\
\hline $\mathrm{CDKN} 2 \mathrm{C}$ & 1416868_at & & & & & & $\mathrm{D}$ & & & \\
\hline CEBPB & 1427844_a_at & & & $\mathrm{D}$ & & $\mathrm{D}$ & & & & \\
\hline CEBPD & 1423233_at & & & & & $\mathrm{D}$ & & I & I & I \\
\hline CENPF & 1427161_at & & & & & & & & & I \\
\hline CFLAR & 1424996_at & & & & & & & & & I \\
\hline CHEK1 & 1439208_at & & & & & & & & & $\mathrm{D}$ \\
\hline CKAP2 & 1434748_at & & & & & I & I & & I & I \\
\hline CLCF1 & $\begin{array}{l}\text { 1437270_a_at, } \\
\text { 1450262_at }\end{array}$ & & & $\mathrm{D}$ & & & & & & I \\
\hline CLU & $\begin{array}{l}\text { 1418626_a_at, } \\
\text { 1437458_x_at, } \\
\text { 1437689_x_at, } \\
\text { 1454849_x_at }\end{array}$ & & & & & & & I & I & I \\
\hline CNN2 & 1450981_at & & & & & & & & I & I \\
\hline CNP & $\begin{array}{l}\text { 1418980_a_at, } \\
\text { 1437341_x_at }\end{array}$ & & & & & & & & I & I \\
\hline CNTF & 1426327_s_at & & & & & & & & & $\mathrm{D}$ \\
\hline COPS5 & 1460171_at & & & & & & & & & $\mathrm{D}$ \\
\hline CR1 & 1422563_at & & & & & & & & & $\mathrm{D}$ \\
\hline CREB3L1 & 1419295_at & & & & & & & & I & I \\
\hline CRK & $\begin{array}{l}\text { 1416201_at, } \\
\text { 1448248_at }\end{array}$ & & & $\mathrm{D}$ & & & I & & I & I \\
\hline
\end{tabular}


Table 1: Continued.

\begin{tabular}{|c|c|c|c|c|c|c|c|c|c|c|}
\hline & & & $3 \mathrm{~h}$ & & & $24 \mathrm{~h}$ & & & $72 \mathrm{~h}$ & \\
\hline Symbol & Probe set ID & Gy & CDDP & $\begin{array}{l}\text { Gy }+ \\
\text { CDDP }\end{array}$ & Gy & CDDP & $\begin{array}{l}\text { Gy }+ \\
\text { CDDP }\end{array}$ & Gy & CDDP & $\begin{array}{l}\text { Gy + } \\
\text { CDDP }\end{array}$ \\
\hline CROP & $\begin{array}{l}\text { 1424802_a_at, } \\
\text { 1451485_at }\end{array}$ & & & I & & I & & & & $\mathrm{D}$ \\
\hline CRYAB & $\begin{array}{l}\text { 1416455_a_at, } \\
1434369 \text { _a_at }\end{array}$ & & & I & & & & & I & I \\
\hline CSF1 & $\begin{array}{l}\text { 1425154_a_at, } \\
\text { 1425155_x_at, } \\
\text { 1448914_a_at, } \\
\text { 1460220_a_at }\end{array}$ & & & D & & & & & I & I \\
\hline CSF2 & 1427429_at & & & & & & & & & I \\
\hline CSNK2A1 & $\begin{array}{l}\text { 1419034_at, } \\
\text { 1419035_s_at, } \\
\text { 1419036_at, } \\
\text { 1419038_a_at }\end{array}$ & & & & & & & & $\mathrm{D}$ & $\mathrm{D}$ \\
\hline CST3 & 1426195_a_at & & & & & & & I & & \\
\hline CTCF & $\begin{array}{l}\text { 1418330_at, } \\
\text { 1449042_at }\end{array}$ & $\mathrm{D}$ & & & & & & & & $\mathrm{D}$ \\
\hline CTGF & 1416953_at & & & & & & & I & I & I \\
\hline CTNNA1 & $\begin{array}{l}\text { 1437807_x_at, } \\
\text { 1448149_at }\end{array}$ & & & & & & & & & I \\
\hline CTSB & $\begin{array}{l}\text { 1417490_at, } \\
\text { 1417491_at, } \\
\text { 1417492_at }\end{array}$ & & & & & & & & I & I \\
\hline CTSD & 1448118_a_at & & & & & & & & I & I \\
\hline CTTN & $\begin{array}{l}\text { 1421313_s_at, } \\
\text { 1421315_s_at, } \\
\text { 1423917_a_at, } \\
\text { 1433908_a_at }\end{array}$ & & & & & & & & I & I \\
\hline CUL3 & 1434717_at & & & & & & & $\mathrm{D}$ & & \\
\hline CUL5 & 1428287_at & & & & & I & & & & \\
\hline CX3CL1 & 1415803_at & & & & & & & & & I \\
\hline CXCL12 & $\begin{array}{l}\text { 1417574_at, } \\
\text { 1448823_at }\end{array}$ & & & & & $\mathrm{D}$ & $\mathrm{D}$ & & & \\
\hline CXCL2 & $\begin{array}{l}\text { 1419209_at, } \\
\text { 1441855_x_at, } \\
\text { 1457644_s_at }\end{array}$ & & & & & $\mathrm{D}$ & & I & I & I \\
\hline CXCR7 & 1417625_s_at & & & & & D & $\mathrm{D}$ & & I & I \\
\hline CYB5A & 1416727_a_at & & & & & & & & & I \\
\hline CYB5R3 & $\begin{array}{l}\text { 1422185_a_at, } \\
\text { 1422186_s_at, } \\
\text { 1425329_a_at }\end{array}$ & & & & & & & & I & I \\
\hline СYBA & 1454268_a_at & & & & & & & & I & \\
\hline CYLD & 1429617_at & & & & & & & & & I \\
\hline CYR61 & $\begin{array}{l}\text { 1416039_x_at, } \\
\text { 1438133_a_at, } \\
\text { 1442340_x_at, } \\
\text { 1457823_at }\end{array}$ & & & & & & & I & I & I \\
\hline DAB2 & $\begin{array}{l}\text { 1420498_a_at, } \\
\text { 1423805_at, } \\
\text { 1429693_at }\end{array}$ & & & & & & & & I & I \\
\hline DAP & $\begin{array}{l}\text { 1423790_at, } \\
1451112 \text { s_at }\end{array}$ & & & & & $\mathrm{D}$ & $\mathrm{D}$ & I & I & I \\
\hline DAXX & 1419026_at & & & & & & & D & I & \\
\hline
\end{tabular}


TABle 1: Continued.

\begin{tabular}{|c|c|c|c|c|c|c|c|c|c|c|}
\hline & & & $3 \mathrm{~h}$ & & & $24 \mathrm{~h}$ & & & $72 \mathrm{~h}$ & \\
\hline Symbol & Probe set ID & Gy & CDDP & $\begin{array}{l}\text { Gy }+ \\
\text { CDDP }\end{array}$ & Gy & CDDP & $\begin{array}{l}\text { Gy }+ \\
\text { CDDP }\end{array}$ & Gy & CDDP & $\begin{array}{l}\text { Gy }+ \\
\text { CDDP }\end{array}$ \\
\hline DCN & 1449368_at & & & & & & & & & I \\
\hline DDIT3 & 1417516_at & & & & & & & & & $\mathrm{D}$ \\
\hline DDIT4 & 1428306_at & & & & & & & I & I & I \\
\hline DDR1 & $\begin{array}{l}\text { 1415797_at, } \\
\text { 1415798_at, } \\
\text { 1456226_x_at }\end{array}$ & & & $\mathrm{D}$ & & & & & I & I \\
\hline DDX5 & 1419653_a_at & I & & & & & & & & \\
\hline DDX58 & $\begin{array}{l}\text { 1436562_at, } \\
\text { 1456890_at }\end{array}$ & & & & & & & $\mathrm{D}$ & I & $\mathrm{D}$ \\
\hline DHCR24 & 1451895_a_at & & & & & & & & I & I \\
\hline DKK3 & $\begin{array}{l}\text { 1417312_at, } \\
\text { 1448669_at }\end{array}$ & & & & & $\mathrm{D}$ & $\mathrm{D}$ & I & I & I \\
\hline DLC1 & $\begin{array}{l}\text { 1436173_at, } \\
1460602 \text { at }\end{array}$ & & & & & & & & & I \\
\hline DLX2 & 1448877_at & & & & & $\mathrm{D}$ & & & & \\
\hline DNAJC15 & 1416910_at & & & & & & & & & $\mathrm{D}$ \\
\hline DNM1L & $\begin{array}{l}\text { 1428086_at, } \\
\text { 1452638_s_at }\end{array}$ & & & & & I & & & & \\
\hline DTYMK & 1438096_a_at & & & I & & & & & & \\
\hline DUSP14 & 1431422_a_at & & & & & & & & I & I \\
\hline DUSP22 & 1448985_at & & & & & & & & I & \\
\hline DUSP4 & 1428834_at & & & $\mathrm{D}$ & & & & & & \\
\hline DUSP6 & 1415834_at & & & & & & & & & I \\
\hline DUT & 1419270_a_at & & & & & & & & & $\mathrm{D}$ \\
\hline E2F1 & 1417878_at & & & & & & & $\mathrm{D}$ & & \\
\hline ECOP & 1451127_at & & & & & & & $\mathrm{D}$ & & \\
\hline EDA2R & 1440085_at & I & & I & & & & I & I & I \\
\hline EEF1D & $\begin{array}{l}\text { 1439439_x_at, } \\
1449506 \text { _a_at }\end{array}$ & & & & & & & & $\mathrm{D}$ & $\mathrm{D}$ \\
\hline EGR1 & 1417065_at & & & & & & & I & I & I \\
\hline EHD4 & 1449852_a_at & & & & & & & & I & \\
\hline EIF2AK2 & $\begin{array}{l}\text { 1422006_at, } \\
\text { 1440866_at }\end{array}$ & & & & I & I & I & & & \\
\hline EIF4E & 1450908_at & & & & & & & & & $\mathrm{D}$ \\
\hline EIF5A & 1437859_x_at & & & & & & & & & $\mathrm{D}$ \\
\hline ELAVL1 & 1452858_at & & & & & & & & & $\mathrm{D}$ \\
\hline EMILIN2 & 1435264_at & & & & & & & & I & I \\
\hline EMP1 & 1416529_at & & & & & & & I & I & I \\
\hline EMP3 & 1417104_at & & & & & & & I & I & I \\
\hline ENO1 & $\begin{array}{l}\text { 1419022_a_at, } \\
\text { 1419023_x_at }\end{array}$ & & & & & & & I & & \\
\hline EPHA2 & 1421151_a_at & & & & & & & & & I \\
\hline EPHX1 & 1422438_at & & & & & & & I & I & I \\
\hline ERCC3 & 1448497_at & & & & & & & & & I \\
\hline ERCC5 & 1450935_at & & & & & & & & & I \\
\hline ESPL1 & 1433862_at & & & & & & & & & I \\
\hline ETS1 & $\begin{array}{l}\text { 1422027_a_at, } \\
\text { 1426725_s_at, } \\
1452163 \text { at }\end{array}$ & & & $\mathrm{D}$ & & & & & & I \\
\hline
\end{tabular}


Table 1: Continued.

\begin{tabular}{|c|c|c|c|c|c|c|c|c|c|c|}
\hline & & & $3 \mathrm{~h}$ & & & $24 \mathrm{~h}$ & & & $72 \mathrm{~h}$ & \\
\hline Symbol & Probe set ID & Gy & CDDP & $\begin{array}{l}\text { Gy }+ \\
\text { CDDP }\end{array}$ & Gy & CDDP & $\begin{array}{l}\text { Gy }+ \\
\text { CDDP }\end{array}$ & Gy & CDDP & $\begin{array}{l}\mathrm{Gy}+ \\
\mathrm{CDDP}\end{array}$ \\
\hline ETS2 & 1416268_at & & & & & & & $\mathrm{D}$ & & \\
\hline EVI1 & 1438325_at & & & & & $\mathrm{D}$ & $\mathrm{D}$ & & & \\
\hline EWSR1 & 1417238_at & & & & & & & & I & I \\
\hline EXOC2 & 1428470_at & & & & & & & & & I \\
\hline EZR & 1450850_at & & & & & & & & I & I \\
\hline F2R & $\begin{array}{l}\text { 1437308_s_at, } \\
1450852 \text { _s_at }\end{array}$ & & & & & & & & & I \\
\hline F3 & 1417408_at & & & & & & $\mathrm{D}$ & & & \\
\hline FAS & 1460251_at & & & & & & & & & I \\
\hline FASN & 1423828_at & & & & & & & & & I \\
\hline FBL & $\begin{array}{l}\text { 1416684_at, } \\
\text { 1416685_s_at }\end{array}$ & & & & & & & & $\mathrm{D}$ & $\mathrm{D}$ \\
\hline FBN1 & $\begin{array}{l}\text { 1425896_a_at, } \\
\text { 1438870_at, } \\
1460208 \text { at }\end{array}$ & & & & & & & & I & I \\
\hline FDFT1 & $\begin{array}{l}\text { 1438322_x_at, } \\
\text { 1448130_at }\end{array}$ & & & & & & $\mathrm{D}$ & & I & I \\
\hline FEN1 & $\begin{array}{l}\text { 1421731_a_at, } \\
\text { 1436454_x_at }\end{array}$ & & & & & & & $\mathrm{D}$ & $\mathrm{D}$ & $\mathrm{D}$ \\
\hline FGF7 & $\begin{array}{l}\text { 1422243_at, } \\
\text { 1438405_at }\end{array}$ & & & & & & & I & I & I \\
\hline FGFR1 & 1424050_s_at & & & $\mathrm{D}$ & & & & & I & I \\
\hline FHL2 & 1419184_a_at & & & $\mathrm{D}$ & & & & & I & I \\
\hline FKBP1B & 1449429_at & & & & & & & & I & I \\
\hline FLT3LG & 1422115_a_at & & & & & & & & I & \\
\hline FN1 & 1437218_at & & & & & & & & & I \\
\hline FOS & 1423100_at & & & & & & & & I & I \\
\hline FOSL1 & $\begin{array}{l}\text { 1417487_at, } \\
\text { 1417488_at }\end{array}$ & & & & & & & I & I & I \\
\hline FOXM1 & $\begin{array}{l}\text { 1417748_x_at, } \\
\text { 1448833_at, } \\
\text { 1448834_at, } \\
\text { 1453107_s_at }\end{array}$ & & & & & & & & I & I \\
\hline FOXO1 & 1416982_at & & & & & & $\mathrm{D}$ & & & \\
\hline FOXP1 & $\begin{array}{l}\text { 1421141_a_at, } \\
\text { 1421142_s_at, } \\
\text { 1435222_at }\end{array}$ & & & & & & $\mathrm{D}$ & & & \\
\hline FST & $\begin{array}{l}\text { 1421365_at, } \\
\text { 1434458_at }\end{array}$ & & & & I & & I & I & & I \\
\hline FSTL1 & $\begin{array}{l}\text { 1416221_at, } \\
\text { 1448259_at }\end{array}$ & & & & & & & I & I & I \\
\hline FTH1 & 1427021_s_at & & & & & & & I & & \\
\hline FUBP1 & $\begin{array}{l}\text { 1433482_a_at, } \\
\text { 1433640_at }\end{array}$ & & & & & & I & & $\mathrm{D}$ & $\mathrm{D}$ \\
\hline FUS & $\begin{array}{l}\text { 1451285_at, } \\
\text { 1455831_at }\end{array}$ & & & I & & & & $\mathrm{D}$ & & \\
\hline FXN & 1427282_a_at & & & & & & & & & $\mathrm{D}$ \\
\hline
\end{tabular}


Table 1: Continued.

\begin{tabular}{|c|c|c|c|c|c|c|c|c|c|c|}
\hline & & & $3 \mathrm{~h}$ & & & $24 \mathrm{~h}$ & & & $72 \mathrm{~h}$ & \\
\hline Symbol & Probe set ID & Gy & CDDP & $\begin{array}{l}\text { Gy }+ \\
\text { CDDP }\end{array}$ & Gy & CDDP & $\begin{array}{l}\text { Gy }+ \\
\text { CDDP }\end{array}$ & Gy & CDDP & $\begin{array}{l}\mathrm{Gy}+ \\
\mathrm{CDDP}\end{array}$ \\
\hline FXR1 & $\begin{array}{l}\text { 1417598_a_at, } \\
\text { 1442059_at, } \\
\text { 1452247_at }\end{array}$ & & & & & I & & & & \\
\hline FYN & 1448765_at & & & & & & & & & I \\
\hline G2E3 & $\begin{array}{l}\text { 1434699_at, } \\
\text { 1455355_at }\end{array}$ & & & & & & & & I & I \\
\hline G6PD & 1448354_at & & & & & & & & I & I \\
\hline GABPA & 1450665_at & & & & & & & & & $\mathrm{D}$ \\
\hline GADD45A & 1449519_at & & & & & & & $\mathrm{D}$ & $\mathrm{D}$ & $\mathrm{D}$ \\
\hline GAS1 & $\begin{array}{l}\text { 1416855_at, } \\
\text { 1448494_at }\end{array}$ & $\mathrm{D}$ & & & $\mathrm{D}$ & $\mathrm{D}$ & $\mathrm{D}$ & & $\mathrm{D}$ & $\mathrm{D}$ \\
\hline GATAD2A & $\begin{array}{l}\text { 1423992_at, } \\
\text { 1451197_s_at, } \\
\text { 1451198_at, } \\
\text { 1455505_at }\end{array}$ & & & $\mathrm{D}$ & & & & & & I \\
\hline GDF15 & 1418949_at & I & & & & & & & I & \\
\hline GDNF & 1419080_at & & & & & & & & & I \\
\hline GFRA1 & 1450440_at & & & & & & & & I & I \\
\hline GHR & $\begin{array}{l}\text { 1417962_s_at, } \\
1451501 \text { a_at }\end{array}$ & & & & & & & & I & I \\
\hline GJA1 & $\begin{array}{l}\text { 1415800_at, } \\
\text { 1415801_at, } \\
\text { 1437992_x_at, } \\
\text { 1437992_x_at, } \\
\text { 1438650_x_at, } \\
\text { 1438945_x_at, } \\
\text { 1438973_x_at }\end{array}$ & & & & & $\mathrm{D}$ & $\mathrm{D}$ & I & & I \\
\hline GLIPR1 & 1424927_at & & & & & & & I & I & I \\
\hline GLRX & $\begin{array}{l}\text { 1416592_at, } \\
1416593 \_a t\end{array}$ & & & & & & & & I & I \\
\hline GNA12 & $\begin{array}{l}\text { 1421026_at, } \\
\text { 1450097_s_at }\end{array}$ & & & & & & & & & $\mathrm{D}$ \\
\hline GNA13 & $\begin{array}{l}\text { 1422556_at, } \\
\text { 1433749_at, } \\
\text { 1450656_at, } \\
\text { 1453470_a_at, } \\
\text { 1460317_s_at }\end{array}$ & & & $\mathrm{D}$ & & & & $\mathrm{D}$ & & \\
\hline GNPNAT1 & 1423158_at & & & & & & & & & $\mathrm{D}$ \\
\hline GPI & $\begin{array}{l}\text { 1420997_a_at, } \\
1434814 \text { _x_at, } \\
1450081 \text { x_at }\end{array}$ & & & & & & & & & I \\
\hline GPX1 & 1460671_at & & & & & & & & & I \\
\hline GRN & 1448148_at & & & & & & & & I & \\
\hline GSK3B & $\begin{array}{l}\text { 1437001_at, } \\
1451020 \_a t, \\
1454958 \_a t\end{array}$ & & & & & & & & $\mathrm{D}$ & $\mathrm{D}$ \\
\hline GSN & $\begin{array}{l}\text { 1415812_at, } \\
\text { 1436991_x_at, } \\
\text { 1437171_x_at, } \\
\text { 1456312_x_at }\end{array}$ & & & & & & & & I & I \\
\hline
\end{tabular}


TABle 1: Continued.

\begin{tabular}{|c|c|c|c|c|c|c|c|c|c|c|}
\hline & & & $3 \mathrm{~h}$ & & & $24 \mathrm{~h}$ & & & $72 \mathrm{~h}$ & \\
\hline Symbol & Probe set ID & Gy & CDDP & $\begin{array}{l}\text { Gy }+ \\
\text { CDDP }\end{array}$ & Gy & CDDP & $\begin{array}{l}\text { Gy }+ \\
\text { CDDP }\end{array}$ & Gy & CDDP & $\begin{array}{l}\text { Gy + } \\
\text { CDDP }\end{array}$ \\
\hline GSPT1 & $\begin{array}{l}\text { 1426736_at, } \\
\text { 1452168_x_at }\end{array}$ & & & & & & & & & $\mathrm{D}$ \\
\hline GSTM1 & 1416411_at & & & & & & & & I & \\
\hline GSTM5 & 1448330_at & & & & & & $\mathrm{D}$ & & & \\
\hline HBEGF & 1418349_at & & & $\mathrm{D}$ & & & & & & \\
\hline HELLS & 1417541_at & & & & & I & & & & \\
\hline HIP1 & 1434557_at & & & $\mathrm{D}$ & & & & & & \\
\hline HIPK1 & 1424540_at & & & $\mathrm{D}$ & & & & & & \\
\hline HIST1H1C & $\begin{array}{l}\text { 1416101_a_at, } \\
\text { 1436994_a_at }\end{array}$ & & & & & $\mathrm{D}$ & $\mathrm{D}$ & I & I & \\
\hline HK1 & 1420901_a_at & & & & & & & & I & I \\
\hline HK2 & 1422612_at & & & & & & & & & I \\
\hline HMGA1 & 1416184_s_at & & & & & & & I & I & I \\
\hline HMGA2 & $\begin{array}{l}\text { 1422851_at, } \\
\text { 1450780_s_at, } \\
\text { 1450781_at }\end{array}$ & & & & & & & I & I & I \\
\hline HMGB1L1 & $\begin{array}{l}\text { 1425048_a_at, } \\
\text { 1435324_x_at, } \\
\text { 1439463_x_at, } \\
\text { 1448235_s_at }\end{array}$ & & & & & & & & $\mathrm{D}$ & $\mathrm{D}$ \\
\hline HMGN1 & 1455897_x_at & & & & & & & & $\mathrm{D}$ & \\
\hline HMMR & $\begin{array}{l}\text { 1425815_a_at, } \\
\text { 1427541_x_at, } \\
\text { 1450156_a_at, } \\
\text { 1450157_a_at }\end{array}$ & & & & & I & & & I & I \\
\hline HMOX1 & 1448239_at & & & & & & & & & $\mathrm{D}$ \\
\hline HNRNPA1 & $\begin{array}{l}\text { 1423531_a_at, } \\
\text { 1430019_a_at, } \\
1430020 \text { _atat }\end{array}$ & & & & & & & $\mathrm{D}$ & $\mathrm{D}$ & $\mathrm{D}$ \\
\hline HOXA7 & 1449499_at & & & & & & & & & $\mathrm{D}$ \\
\hline HSH2D & 1442130_at & & & & & & & & I & I \\
\hline HSP90AA1 & $\begin{array}{l}\text { 1426645_at, } \\
\text { 1437497_a_at, } \\
\text { 1438902_a_at }\end{array}$ & & & I & & I & I & & & \\
\hline HSP90AB1 & $\begin{array}{l}\text { 1416364_at, } \\
\text { 1416365_at }\end{array}$ & & & & & & & & & I \\
\hline HSPA1B & 1427127_x_at & & & & & & $\mathrm{D}$ & & & \\
\hline HSPA5 & $\begin{array}{l}\text { 1416064_a_at, } \\
\text { 1427464_s_at, } \\
\text { 1447824_x_at }\end{array}$ & & & & & & & & $\mathrm{D}$ & $\mathrm{D}$ \\
\hline HSPB1 & $\begin{array}{l}\text { 1422943_a_at, } \\
\text { 1425964_x_at }\end{array}$ & & & $\mathrm{D}$ & & & & I & I & I \\
\hline HSPB8 & 1417014_at & & & & & & & & & $\mathrm{D}$ \\
\hline HTATIP2 & 1451814_a_at & & & & & & & & & I \\
\hline HUWE1 & 1415703_at & & & & & & & & & $\mathrm{D}$ \\
\hline ID1 & 1425895_a_at & & & & & & & & & I \\
\hline ID2 & 1422537_a_at & & & & & & D & & & \\
\hline IER3 & 1419647_a_at & & & & & & & I & I & I \\
\hline IFI16 & $\begin{array}{l}\text { 1419603_at, } \\
\text { 1452349_x_at }\end{array}$ & & & & & I & & $\mathrm{D}$ & & \\
\hline
\end{tabular}


Table 1: Continued.

\begin{tabular}{|c|c|c|c|c|c|c|c|c|c|c|}
\hline & & & $3 \mathrm{~h}$ & & & $24 \mathrm{~h}$ & & & $72 \mathrm{~h}$ & \\
\hline Symbol & Probe set ID & Gy & CDDP & $\begin{array}{l}\text { Gy }+ \\
\text { CDDP }\end{array}$ & Gy & CDDP & $\begin{array}{l}\text { Gy }+ \\
\text { CDDP }\end{array}$ & Gy & CDDP & $\begin{array}{l}\text { Gy }+ \\
\text { CDDP }\end{array}$ \\
\hline IFI202B & $\begin{array}{l}\text { 1421551_s_at, } \\
\text { 1457666_s_at }\end{array}$ & & & & I & I & I & & & \\
\hline IFIH1 & 1426276_at & & & & & I & & $\mathrm{D}$ & & \\
\hline IFNAR2 & 1451462_a_at & & & & & & & & & I \\
\hline IGFBP4 & $\begin{array}{l}\text { 1421992_aaat, } \\
\text { 1423756_s_at, } \\
\text { 1423757_x_at, } \\
\text { 1437405_a_at, } \\
\text { 1437406_x_at }\end{array}$ & & & & & & & & $\mathrm{D}$ & $\mathrm{D}$ \\
\hline IGFBP5 & $\begin{array}{l}\text { 1422313_a_at, } \\
\text { 1452114_s_at }\end{array}$ & & & & & $\mathrm{D}$ & D & & $\mathrm{D}$ & $\mathrm{D}$ \\
\hline IGFBP7 & $\begin{array}{l}\text { 1423584_at, } \\
\text { 1423585_at }\end{array}$ & & & & & $\mathrm{D}$ & & & I & I \\
\hline IKBKG & 1454690_at & & & & & & & & & I \\
\hline IKIP & $\begin{array}{l}\text { 1429065_at, } \\
\text { 1429219_at }\end{array}$ & & & & & & & & I & I \\
\hline IL15 & 1418219_at & & & & & & & & I & \\
\hline IL15RA & 1448681_at & & & & & & & & & I \\
\hline IL18 & 1417932_at & & & & & & & & I & I \\
\hline IL1RL1 & 1422317_a_at & & & & & & & & & I \\
\hline IL6 & 1450297_at & I & & & & & & & I & I \\
\hline INHBA & 1422053_at & & & & & & & I & I & I \\
\hline INPP1 & $\begin{array}{l}\text { 1418045_at, } \\
\text { 1442073_at }\end{array}$ & & & & & & & & I & \\
\hline IRF8 & $\begin{array}{l}\text { 1416714_at, } \\
\text { 1448452_at }\end{array}$ & & & & & & I & & & I \\
\hline IRS1 & 1423104_at & D & & & & & & I & I & I \\
\hline ITGA5 & 1423267_s_at & & & $\mathrm{D}$ & & & & & & \\
\hline ITGB5 & $\begin{array}{l}\text { 1417533_aadat, } \\
\text { 1417534_at, } \\
\text { 1456195_x_at }\end{array}$ & & & & & $\mathrm{D}$ & $\mathrm{D}$ & & & \\
\hline ITM2B & $\begin{array}{l}\text { 1417999_at, } \\
\text { 1418000_a_at }\end{array}$ & & & & & & & & I & I \\
\hline ITPR3 & 1417297_at & & & & & & & & I & I \\
\hline JMJD6 & $\begin{array}{l}\text { 1420056_s_at, } \\
\text { 1454109_a_at }\end{array}$ & & & & & & & & $\mathrm{D}$ & $\mathrm{D}$ \\
\hline JUN & $\begin{array}{l}\text { 1417409_at, } \\
\text { 1448694_at }\end{array}$ & & & & & & & $\mathrm{D}$ & $\mathrm{D}$ & $\mathrm{D}$ \\
\hline KAT2B & $\begin{array}{l}\text { 1434037_s_at, } \\
\text { 1450821_at }\end{array}$ & & & & & & & & I & I \\
\hline KAT5 & $\begin{array}{l}\text { 1433980_at, } \\
\text { 1433981_s_at }\end{array}$ & & & & & & & & & $\mathrm{D}$ \\
\hline KIF1B & 1455182_at & & & & & I & & & & \\
\hline KITLG & $\begin{array}{l}\text { 1415855_at, } \\
\text { 1448117_at }\end{array}$ & & & & & I & & & & I \\
\hline KLF10 & 1416029_at & & & & & & & & I & I \\
\hline KLF4 & $\begin{array}{l}\text { 1417394_at, } \\
\text { 1417395_at }\end{array}$ & & & & & & & & I & I \\
\hline
\end{tabular}


Table 1: Continued.

\begin{tabular}{|c|c|c|c|c|c|c|c|c|c|c|}
\hline & & & $3 \mathrm{~h}$ & & & $24 \mathrm{~h}$ & & & $72 \mathrm{~h}$ & \\
\hline Symbol & Probe set ID & Gy & CDDP & $\begin{array}{l}\text { Gy }+ \\
\text { CDDP }\end{array}$ & Gy & CDDP & $\begin{array}{l}\mathrm{Gy}+ \\
\mathrm{CDDP}\end{array}$ & Gy & CDDP & $\begin{array}{l}\mathrm{Gy}+ \\
\mathrm{CDDP}\end{array}$ \\
\hline KLF6 & $\begin{array}{l}\text { 1418280_at, } \\
\text { 1427742_a_at, } \\
\text { 1447448_s_at }\end{array}$ & $\mathrm{D}$ & $\mathrm{D}$ & $\mathrm{D}$ & I & & & & & \\
\hline LAMP2 & 1416344_at & & & & & & & & I & I \\
\hline LCN2 & 1427747_a_at & & & & & & & I & & I \\
\hline LDLR & 1421821_at & & & & & & $\mathrm{D}$ & & I & I \\
\hline LGALS3 & 1426808_at & & & & & & & & & I \\
\hline LGALS3BP & 1448380_at & & & & & & & & I & I \\
\hline LGALS8 & 1422662_at & & & & & & & & I & \\
\hline LIF & 1421207_at & & & & & & & & & I \\
\hline LIMS1 & 1418232_s_at & & & $\mathrm{D}$ & & & & & & \\
\hline LMNA & $\begin{array}{l}\text { 1421654_a_at, } \\
\text { 1425472_a_at, } \\
\text { 1457670_s_at }\end{array}$ & & & $\mathrm{D}$ & & $\mathrm{D}$ & $\mathrm{D}$ & I & I & I \\
\hline LPAR1 & $\begin{array}{l}\text { 1426110_a_at, } \\
\text { 1448606_at }\end{array}$ & & & & & $\mathrm{D}$ & $\mathrm{D}$ & & & I \\
\hline LRIG1 & $\begin{array}{l}\text { 1434210_s_at, } \\
\text { 1449893_a_at }\end{array}$ & & & & & & & & I & I \\
\hline LTBR & 1416435_at & & & $\mathrm{D}$ & & & & & & \\
\hline MAOA & 1428667_at & & & & & & & I & I & I \\
\hline MAP2K3 & 1451714_a_at & & & & & & & & & I \\
\hline MAP3K12 & 1438908_at & I & & & & & & & & \\
\hline MAP3K4 & 1459800_s_at & & & & & & & & I & I \\
\hline MAP3K7 & 1419988_at & & & & & & & & & I \\
\hline MAPK3 & 1427060_at & & & $\mathrm{D}$ & & & & & & \\
\hline MAPK8 & 1420932_at & & & & & & & & & $\mathrm{D}$ \\
\hline MAPKAP1 & 1417284_at & & & & & & & & I & I \\
\hline MAX & 1423501_at & & & & & & $\mathrm{D}$ & $\mathrm{D}$ & & $\mathrm{D}$ \\
\hline MCF2L & 1434140_at & & & $\mathrm{D}$ & & & & & & \\
\hline MCL1 & 1416880_at & & & $\mathrm{D}$ & & & & & & \\
\hline MCM2 & $\begin{array}{l}\text { 1448777_at, } \\
\text { 1423605_a_at, } \\
\text { 1427718_a_at }\end{array}$ & & & & & & & $\mathrm{D}$ & $\mathrm{D}$ & $\mathrm{D}$ \\
\hline MDM2 & 1427718_a_at & I & & I & & I & I & & I & I \\
\hline MED1 & 1450402_at & & & I & & & & & & \\
\hline MEF2A & $\begin{array}{l}\text { 1427186_a_at, } \\
\text { 1452347_at }\end{array}$ & & & & I & & & & & \\
\hline MET & $\begin{array}{l}\text { 1422990_at, } \\
\text { 1434447_at }\end{array}$ & & & & & & & & I & I \\
\hline MFGE8 & 1420911_a_at & & & & & & & & & I \\
\hline MGP & 1448416_at & & & & & $\mathrm{D}$ & $\mathrm{D}$ & I & & I \\
\hline MGST1 & 1415897_a_at & & & & & & & I & I & I \\
\hline MMP2 & 1416136_at & & & & & & & & & I \\
\hline MMP3 & 1418945_at & & & & & & & I & & I \\
\hline MPG & $\begin{array}{l}\text { 1417571_at, } \\
\text { 1417572_at }\end{array}$ & & & & & & & & I & I \\
\hline MT1E & 1428942_at & & & & & $\mathrm{D}$ & & I & I & I \\
\hline
\end{tabular}


TABle 1: Continued.

\begin{tabular}{|c|c|c|c|c|c|c|c|c|c|c|}
\hline & & & $3 \mathrm{~h}$ & & & $24 \mathrm{~h}$ & & & $72 \mathrm{~h}$ & \\
\hline Symbol & Probe set ID & Gy & CDDP & $\begin{array}{l}\text { Gy }+ \\
\text { CDDP }\end{array}$ & Gy & CDDP & $\begin{array}{l}\text { Gy }+ \\
\text { CDDP }\end{array}$ & Gy & CDDP & $\begin{array}{l}\text { Gy }+ \\
\text { CDDP }\end{array}$ \\
\hline MT1F & 1422557_s_at & & & & & & $\mathrm{D}$ & I & & \\
\hline MTMR6 & 1425485_at & & & & & & & & & I \\
\hline MTPN & 1437457_a_at & & & & I & & & & & \\
\hline MX1 & 1451905_a_at & & & & & & & $\mathrm{D}$ & I & \\
\hline MYC & 1424942_a_at & & & $\mathrm{D}$ & & & & & & \\
\hline MYO6 & 1433942_at & & & & & & & & & I \\
\hline NAMPT & 1417190_at & & & & & & & $\mathrm{D}$ & & $\mathrm{D}$ \\
\hline NCAM1 & 1426864_a_at & & & & & & & & & I \\
\hline NCAPG2 & 1417926_at & & & & & I & & & & \\
\hline NDRG1 & $\begin{array}{l}\text { 1420760_s_at, } \\
\text { 1423413_at, } \\
\text { 1450976_at, } \\
\text { 1456174_x_at }\end{array}$ & & & & & & & $\mathrm{D}$ & $\mathrm{D}$ & $\mathrm{D}$ \\
\hline NDST1 & $\begin{array}{l}\text { 1422044_at, } \\
\text { 1460436_at }\end{array}$ & & & $\mathrm{D}$ & & & & $\mathrm{D}$ & & \\
\hline NDUFAF4 & 1427997_at & & & I & & & & & & \\
\hline NDUFV2 & $\begin{array}{l}\text { 1428179_at, } \\
\text { 1452692_a_at }\end{array}$ & & & & & & & & & I \\
\hline NEDD9 & 1422818_at & & & & & & & & & I \\
\hline NEK2 & $\begin{array}{l}\text { 1417299_at, } \\
\text { 1437580_s_at }\end{array}$ & & & & & & & & I & I \\
\hline NEK6 & $\begin{array}{l}\text { 1423596_at, } \\
\text { 1425850_a_at }\end{array}$ & & & & & & & I & & I \\
\hline NFAT5 & $\begin{array}{l}\text { 1438999_a_at, } \\
\text { 1439805_at }\end{array}$ & & & $\mathrm{D}$ & & & I & & I & I \\
\hline NFIL3 & 1418932_at & & & $\mathrm{D}$ & & & & & & \\
\hline NFKB1 & 1427705_a_at & & & & & & & & & I \\
\hline NFKB2 & 1425902_a_at & & & & & & I & & & \\
\hline NFKBIA & $\begin{array}{l}\text { 1420088_at, } \\
\text { 1438157_s_at, } \\
\text { 1448306_at, } \\
\text { 1449731_s_at }\end{array}$ & & & & & & & I & I & I \\
\hline NFKBIZ & $\begin{array}{l}\text { 1417483_at, } \\
\text { 1448728_a_at, } \\
\text { 1457404_at }\end{array}$ & & & & & & & I & I & I \\
\hline NGF & 1419675_at & & & $\mathrm{D}$ & & & & & & \\
\hline NME1 & 1424110_a_at & & & & & & & & & $\mathrm{D}$ \\
\hline NOD1 & 1454733_at & & & & & & & & I & I \\
\hline NOTCH2 & 1455556_at & & & & & & $\mathrm{D}$ & & & \\
\hline NP & $\begin{array}{l}\text { 1416530_a_at, } \\
\text { 1453299_a_at }\end{array}$ & & & & & & & & I & I \\
\hline NQO1 & 1423627_at & & & & & & & & I & I \\
\hline NQO2 & $\begin{array}{l}\text { 1449983_a_at, } \\
\text { 1455590_at }\end{array}$ & & & & & & & & I & I \\
\hline NR2F1 & 1418157_at & & & & & & $\mathrm{D}$ & & & \\
\hline NR3C1 & $\begin{array}{l}\text { 1421867_at, } \\
\text { 1457635_s_at, } \\
\text { 1460303_at }\end{array}$ & & & & & & & & I & I \\
\hline NR4A1 & 1416505_at & & & $\mathrm{D}$ & & & & & & \\
\hline
\end{tabular}


TABle 1: Continued.

\begin{tabular}{|c|c|c|c|c|c|c|c|c|c|c|}
\hline & & & $3 \mathrm{~h}$ & & & $24 \mathrm{~h}$ & & & $72 \mathrm{~h}$ & \\
\hline Symbol & Probe set ID & Gy & CDDP & $\begin{array}{l}\text { Gy }+ \\
\text { CDDP }\end{array}$ & Gy & CDDP & $\begin{array}{l}\text { Gy }+ \\
\text { CDDP }\end{array}$ & Gy & CDDP & $\begin{array}{l}\text { Gy + } \\
\text { CDDP }\end{array}$ \\
\hline NRF1 & 1434627_at & & & & & & & & & $\mathrm{D}$ \\
\hline NRP1 & 1418084_at & & & & & & & & & I \\
\hline NT5C3 & 1451050_at & & & & & & & & I & I \\
\hline NTRK3 & 1433825_at & & & & $\mathrm{D}$ & $\mathrm{D}$ & $\mathrm{D}$ & & & \\
\hline NUAK2 & 1429049_at & & & & & & & & & I \\
\hline NUPR1 & 1419665_a_at & & & & & & I & & & \\
\hline OAS1 & 1424775_at & & & & & & & & I & \\
\hline OAS1B & 1425119_at & & & & & & & $\mathrm{D}$ & & \\
\hline OAS3 & 1425374_at & & & & & & & $\mathrm{D}$ & I & I \\
\hline ODC1 & 1437711_x_at & & & & & & & & $\mathrm{D}$ & \\
\hline OSGIN1 & 1424022_at & & & $\mathrm{D}$ & & & & & & \\
\hline P2RX4 & $\begin{array}{l}\text { 1425525_a_at, } \\
\text { 1452527_a_at }\end{array}$ & & & & & & & & I & I \\
\hline P2RX7 & 1439787_at & & & & & & & & I & \\
\hline PA2G4 & $\begin{array}{l}\text { 1420142_s_at, } \\
\text { 1423060_at, } \\
\text { 1435372_a_at }\end{array}$ & & & $\mathrm{D}$ & & & & & $\mathrm{D}$ & $\mathrm{D}$ \\
\hline PAFAH1B1 & 1460199_a_at & & & & & & & $\mathrm{D}$ & & $\mathrm{D}$ \\
\hline PAK1 & $\begin{array}{l}\text { 1420980_at, } \\
\text { 1450070_s_at }\end{array}$ & & & $\mathrm{D}$ & & & & & & I \\
\hline PAK3 & $\begin{array}{l}\text { 1435486_at, } \\
\text { 1437318_at }\end{array}$ & & & $\mathrm{D}$ & & I & & & & \\
\hline PALLD & $\begin{array}{l}\text { 1427228_at, } \\
\text { 1433768_at }\end{array}$ & & & $\mathrm{D}$ & & & & & & I \\
\hline PARK7 & $\begin{array}{l}\text { 1416526_a_at, } \\
\text { 1456194_a_at }\end{array}$ & & & & & & & & $\mathrm{D}$ & $\mathrm{D}$ \\
\hline PARVA & 1431375_s_at & & & & & & & & I & I \\
\hline PARVB & 1438672_at & & & & & & & & & I \\
\hline PAWR & 1426910_at & & & $\mathrm{D}$ & & & & & & I \\
\hline PCNA & 1417947_at & & & & & & & & $\mathrm{D}$ & $\mathrm{D}$ \\
\hline PDCD2 & 1423534_at & & & & & & & & & $\mathrm{D}$ \\
\hline PDGFRA & 1421917_at & & & & & D & $\mathrm{D}$ & & & \\
\hline PDGFRB & $\begin{array}{l}\text { 1417148_at, } \\
\text { 1436970_a_at }\end{array}$ & & & $\mathrm{D}$ & $\mathrm{D}$ & $\mathrm{D}$ & D & & & \\
\hline PEA15 & 1416407_at & & & & & & & & & I \\
\hline PHLDA1 & 1418835_at & & & & & & & I & I & I \\
\hline PIK3CA & 1460326_at & & & & & I & & & & \\
\hline PIK3R2 & 1418463_at & & & & & & & & & I \\
\hline PITPNA & $\begin{array}{l}\text { 1423282_at, } \\
\text { 1423283_at }\end{array}$ & & & & & & & & I & I \\
\hline PKN2 & $\begin{array}{l}\text { 1437295_at, } \\
\text { 1437296_at }\end{array}$ & & & & & I & & & & \\
\hline PLAC8 & 1451335_at & & & & & & & & I & I \\
\hline PLAT & 1415806_at & & & & & & & & I & I \\
\hline PLAUR & 1452521_a_at & & & & & & & & & I \\
\hline
\end{tabular}


TABle 1: Continued.

\begin{tabular}{|c|c|c|c|c|c|c|c|c|c|c|}
\hline & & & $3 \mathrm{~h}$ & & & $24 \mathrm{~h}$ & & & $72 \mathrm{~h}$ & \\
\hline Symbol & Probe set ID & Gy & CDDP & $\begin{array}{l}\text { Gy }+ \\
\text { CDDP }\end{array}$ & Gy & CDDP & $\begin{array}{l}\text { Gy }+ \\
\text { CDDP }\end{array}$ & Gy & CDDP & $\begin{array}{l}\text { Gy }+ \\
\text { CDDP }\end{array}$ \\
\hline PLD1 & 1437113_s_at & & & & & & & & I & I \\
\hline PLD2 & 1417237_at & & & & & & & & I & \\
\hline PLEKHF1 & 1424671_at & & & & & & & & I & I \\
\hline PLK1 & 1448191_at & & & $\mathrm{D}$ & & & & I & I & I \\
\hline PLK3 & 1434496_at & & & & & & & & I & I \\
\hline PLSCR1 & $\begin{array}{l}\text { 1429527_a_at, } \\
\text { 1453181_x_at }\end{array}$ & & & & & & & & I & I \\
\hline PLSCR3 & 1449020_at & & & & & & & & I & \\
\hline PMEPA1 & $\begin{array}{l}\text { 1422706_at, } \\
\text { 1452295_at }\end{array}$ & & & $\mathrm{D}$ & & $\mathrm{D}$ & D & & & \\
\hline PML & $\begin{array}{l}\text { 1448757_at, } \\
\text { 1456103_at }\end{array}$ & & & & & & & $\mathrm{D}$ & I & \\
\hline PNKP & 1416378_at & & & & & & & & I & I \\
\hline PNPT1 & 1452676_a_at & & & & & & & $\mathrm{D}$ & & \\
\hline POLK & 1449483_at & & & & & & & & I & I \\
\hline PPID & 1417057_a_at & & & & & & & & $\mathrm{D}$ & $\mathrm{D}$ \\
\hline PPM1A & $\begin{array}{l}\text { 1429501_s_at, } \\
\text { 1451943_a_at }\end{array}$ & & & & & & & & & $\mathrm{D}$ \\
\hline PPM1F & 1454934_at & & & & & & & & & I \\
\hline PPP1R13L & 1459592_a_at & & & $\mathrm{D}$ & & & & & & \\
\hline PPP1R15A & 1448325_at & & & & & & & & $\mathrm{D}$ & $\mathrm{D}$ \\
\hline PPP2R2A & $\begin{array}{l}\text { 1437730_at, } \\
\text { 1453260_a_at }\end{array}$ & & & & & & & $\mathrm{D}$ & & $\mathrm{D}$ \\
\hline PRDX5 & 1416381_a_at & & & & & & & & & I \\
\hline PRKAR2B & $\begin{array}{l}\text { 1438664_at, } \\
\text { 1456475_s_at }\end{array}$ & & & & & & & & & I \\
\hline PRKCA & 1450945_at & & & & & & & & & I \\
\hline PRKD1 & 1447623_s_at & & & & & & & & & I \\
\hline PRMT2 & 1416844_at & & & & & & & & I & \\
\hline PRPF19 & 1460633_at & & & & & & D & & & \\
\hline PRR13 & 1423686_a_at & & & & & & I & & I & I \\
\hline PSENEN & 1415679_at & & & & & & & & & $\mathrm{D}$ \\
\hline PSIP1 & $\begin{array}{l}\text { 1417166_at, } \\
\text { 1460403_at }\end{array}$ & & & & I & I & I & & & \\
\hline PSMG2 & $\begin{array}{l}\text { 1425373_a_at, } \\
\text { 1448212_at }\end{array}$ & & & & & & & & & $\mathrm{D}$ \\
\hline PTGR1 & 1417777_at & & & & & & & I & I & I \\
\hline PTGS1 & 1436448_a_at & & & & & & & & & I \\
\hline PTGS2 & $\begin{array}{l}\text { 1417262_at, } \\
\text { 1417263_at }\end{array}$ & & & & & & & I & I & I \\
\hline PTMA & 1423455_at & & & & & & & & $\mathrm{D}$ & $\mathrm{D}$ \\
\hline PTPN1 & 1438670_at & & & $\mathrm{D}$ & & & & & & \\
\hline PTPRA & 1425340_a_at & & & & & & & & I & I \\
\hline PTPRE & 1418540_a_at & & & & & & & & I & \\
\hline PTPRG & 1434360_s_at & & & & & D & $\mathrm{D}$ & & & \\
\hline PTRH2 & 1451845_a_at & & & & & & & $\mathrm{D}$ & $\mathrm{D}$ & $\mathrm{D}$ \\
\hline
\end{tabular}


TABle 1: Continued.

\begin{tabular}{|c|c|c|c|c|c|c|c|c|c|c|}
\hline & & & $3 \mathrm{~h}$ & & & $24 \mathrm{~h}$ & & & $72 \mathrm{~h}$ & \\
\hline Symbol & Probe set ID & Gy & CDDP & $\begin{array}{l}\text { Gy }+ \\
\text { CDDP }\end{array}$ & Gy & CDDP & $\begin{array}{l}\text { Gy }+ \\
\text { CDDP }\end{array}$ & Gy & CDDP & $\begin{array}{l}\text { Gy + } \\
\text { CDDP }\end{array}$ \\
\hline PTTG1 & $\begin{array}{l}\text { 1419620_at, } \\
\text { 1424105_a_at, } \\
\text { 1438390_s_at }\end{array}$ & & & & & & & I & I & I \\
\hline PXN & $\begin{array}{l}\text { 1424027_at, } \\
\text { 1456135_s_at }\end{array}$ & & & & & & & & I & I \\
\hline QARS & $\begin{array}{l}\text { 1423712_a_at, } \\
\text { 1456726_x_at }\end{array}$ & & & & & & & & I & I \\
\hline QKI & $\begin{array}{l}\text { 1417073_a_at, } \\
\text { 1425597_a_at, } \\
\text { 1429318_a_at, } \\
\text { 1451179_a_at }\end{array}$ & & & & & & $\mathrm{D}$ & $\mathrm{D}$ & $\mathrm{D}$ & $\mathrm{D}$ \\
\hline RABGGTB & 1419553_a_at & & & & & & & & & I \\
\hline RAD18 & 1451928_a_at & & & & & I & & & & \\
\hline RAD21 & 1416162_at & & & $\mathrm{D}$ & & & & & & \\
\hline RAD54L & 1450862_at & & & & & I & & & I & I \\
\hline RALB & 1417744_a_at & & & & & & & & & I \\
\hline RARG & $\begin{array}{l}\text { 1419415_a_at, } \\
\text { 1419416_a_at }\end{array}$ & & & $\mathrm{D}$ & & & & & & \\
\hline RASA1 & $\begin{array}{l}\text { 1426476_at, } \\
\text { 1426477_at }\end{array}$ & & & & & & & & & I \\
\hline RASSF1 & $\begin{array}{l}\text { 1441737_s_at, } \\
\text { 1448855_at }\end{array}$ & & & & & & I & & & \\
\hline RASSF5 & 1422637_at & & & & & & & & & I \\
\hline RB1 & 1417850_at & & & & & I & & & & \\
\hline RBBP4 & $\begin{array}{l}\text { 1434892_x_at, } \\
\text { 1454791_a_at, } \\
\text { 1454875_a_at }\end{array}$ & & & & & & & $\mathrm{D}$ & $\mathrm{D}$ & $\mathrm{D}$ \\
\hline RBBP6 & 1425114_at & & & & & & & & & $\mathrm{D}$ \\
\hline RBL1 & $\begin{array}{l}\text { 1424156_at, } \\
\text { 1425166_at }\end{array}$ & & & & & & & $\mathrm{D}$ & & $\mathrm{D}$ \\
\hline RBP1 & 1448754_at & & & & & & & I & I & I \\
\hline RCAN2 & 1421425_a_at & & & & & & & & & I \\
\hline RECK & 1450784_at & & & & & & & & I & \\
\hline RFC1 & $\begin{array}{l}\text { 1418342_at, } \\
\text { 1449050_at, } \\
\text { 1451920_a_at }\end{array}$ & & & & I & I & I & & & \\
\hline RFK & $\begin{array}{l}\text { 1415737_at, } \\
\text { 1416230_at }\end{array}$ & & & & & & & & $\mathrm{D}$ & $\mathrm{D}$ \\
\hline RFWD2 & 1426913_at & & & & & & & & I & \\
\hline RGS3 & $\begin{array}{l}\text { 1425296_a_at, } \\
\text { 1425701_a_at }\end{array}$ & & & & & & & I & I & I \\
\hline RIPK1 & $\begin{array}{l}\text { 1419508_at, } \\
\text { 1449485_at }\end{array}$ & & & & & & & & I & I \\
\hline RIPK2 & 1450173_at & & & & & & & & & I \\
\hline RNF34 & 1415791_at & & & I & & & & & & \\
\hline ROCK1 & $\begin{array}{l}\text { 1423444_at, } \\
\text { 1423445_at }\end{array}$ & & & & & & I & & & I \\
\hline RPS3 & 1435151_a_at & & & I & & & & & & \\
\hline RPS3A & 1422475_a_at & & & I & & & & I & & \\
\hline RPS6KB1 & 1454956_at & & & & & I & & & & \\
\hline
\end{tabular}


TABle 1: Continued.

\begin{tabular}{|c|c|c|c|c|c|c|c|c|c|c|}
\hline & & & $3 \mathrm{~h}$ & & & $24 \mathrm{~h}$ & & & $72 \mathrm{~h}$ & \\
\hline Symbol & Probe set ID & Gy & $\mathrm{CDDP}$ & $\begin{array}{l}\text { Gy }+ \\
\text { CDDP }\end{array}$ & Gy & CDDP & $\begin{array}{l}\text { Gy }+ \\
\text { CDDP }\end{array}$ & Gy & CDDP & $\begin{array}{l}\text { Gy + } \\
\text { CDDP }\end{array}$ \\
\hline RRAS & 1418448_at & & & & & & & & I & \\
\hline RRAS2 & 1417398_at & & & & & & & & & I \\
\hline RRM2B & 1437476_at & & & & & & & & I & \\
\hline RTN4 & $\begin{array}{l}\text { 1421116_a_at, } \\
\text { 1452649_at }\end{array}$ & & & & & $\mathrm{D}$ & & & & I \\
\hline S100A1 & $\begin{array}{l}\text { 1417421_at, } \\
\text { 1419814_s_at }\end{array}$ & & & & & & & & I & I \\
\hline S100A10 & $\begin{array}{l}\text { 1416762_at, } \\
\text { 1456642_x_at }\end{array}$ & & & & & & & I & I & I \\
\hline S100A4 & 1424542_at & & & & & $\mathrm{D}$ & $\mathrm{D}$ & I & I & I \\
\hline S100A6 & 1421375_a_at & & & & & & & I & & I \\
\hline S1PR1 & 1423571_at & & & D & & & & & I & I \\
\hline S1PR2 & 1428176_at & & & $\mathrm{D}$ & & & & & & \\
\hline S1PR3 & 1438658_a_at & & & & & & & & & I \\
\hline SAT1 & 1420502_at & & & & & & & & & I \\
\hline SCARB1 & $\begin{array}{l}\text { 1416050_a_at, } \\
\text { 1437378_x_at, } \\
1455820 \_ \text {_at }\end{array}$ & & & & & & & & I & I \\
\hline SDC1 & $\begin{array}{l}\text { 1415943_at, } \\
\text { 1415944_at, } \\
\text { 1437279_x_at }\end{array}$ & & & & & $\mathrm{D}$ & $\mathrm{D}$ & & & I \\
\hline SDC4 & 1448793_a_at & & & & & & & & & I \\
\hline SEMA3A & 1449865_at & & & & & & & & & I \\
\hline SENP1 & 1424330_at & & & & & & & & & $\mathrm{D}$ \\
\hline SERBP1 & 1437280_s_at & & & I & & & & & & \\
\hline SERPINE1 & 1419149_at & & & & & $\mathrm{D}$ & D & I & I & I \\
\hline SERPINE2 & 1416666_at & & & & & & & & & I \\
\hline SERPINF1 & $\begin{array}{l}\text { 1416168_at, } \\
\text { 1453724_a_at }\end{array}$ & & & & & & $\mathrm{D}$ & & & \\
\hline SFRP1 & 1448395_at & & & & & & & & & I \\
\hline SFRP2 & 1448201_at & & & & & $\mathrm{D}$ & & & & \\
\hline SFRS5 & 1423130_a_at & & & I & & & & & & \\
\hline SGK1 & 1416041_at & & & & & & & I & I & I \\
\hline SGMS2 & $\begin{array}{l}\text { 1428663_at, } \\
\text { 1429029_at }\end{array}$ & & & & & & & & & I \\
\hline SGPL1 & 1415892_at & & & & & & D & & & \\
\hline SH3BP5 & $\begin{array}{l}\text { 1421922_at, } \\
\text { 1421923_at }\end{array}$ & & & & & & & & I & I \\
\hline SH3GLB1 & $\begin{array}{l}\text { 1418011_a_at, } \\
\text { 1418012_at }\end{array}$ & & & $\mathrm{D}$ & & & & & I & \\
\hline SH3KBP1 & $\begin{array}{l}\text { 1431592_a_at, } \\
\text { 1460337_at }\end{array}$ & & & & & & & & I & I \\
\hline SHISA5 & $\begin{array}{l}\text { 1423986_a_at, } \\
\text { 1437503_a_at }\end{array}$ & & & & & & & & I & I \\
\hline SHPRH & 1452261_at & & & & & I & & & & \\
\hline SIRT7 & 1424238_at & & & & & & & & I & \\
\hline SKIL & 1452214_at & & & & & & & & & I \\
\hline
\end{tabular}


Table 1: Continued.

\begin{tabular}{|c|c|c|c|c|c|c|c|c|c|c|}
\hline & & & $3 \mathrm{~h}$ & & & $24 \mathrm{~h}$ & & & $72 \mathrm{~h}$ & \\
\hline Symbol & Probe set ID & Gy & CDDP & $\begin{array}{l}\mathrm{Gy}+ \\
\mathrm{CDDP}\end{array}$ & Gy & $\mathrm{CDDP}$ & $\begin{array}{l}\mathrm{Gy}+ \\
\mathrm{CDDP}\end{array}$ & Gy & $\mathrm{CDDP}$ & $\begin{array}{l}\text { Gy }+ \\
\text { CDDP }\end{array}$ \\
\hline SLC25A24 & $\begin{array}{l}\text { 1427483_at, } \\
\text { 1452717_at }\end{array}$ & & & & & & & & I & I \\
\hline SLC2A1 & $\begin{array}{l}\text { 1426599_a_at, } \\
\text { 1434773_a_at }\end{array}$ & & & & $\mathrm{D}$ & & & & & I \\
\hline SLC7A11 & 1420413_at & & & & & & & & I & \\
\hline SLK & $\begin{array}{l}\text { 1425977_a_at, } \\
\text { 1449336_a_at }\end{array}$ & & & & & I & & & & \\
\hline SMN1 & 1426596_a_at & & & & & & & & $\mathrm{D}$ & $\mathrm{D}$ \\
\hline SMNDC1 & 1429043_at & & & & & & & & $\mathrm{D}$ & \\
\hline SNRPE & 1451294_s_at & & & & & & & & $\mathrm{D}$ & $\mathrm{D}$ \\
\hline SOCS3 & $\begin{array}{l}\text { 1416576_at, } \\
\text { 1455899_x_at, } \\
\text { 1456212_x_at }\end{array}$ & & & $\mathrm{D}$ & & & & I & I & I \\
\hline SOD2 & $\begin{array}{l}\text { 1417193_at, } \\
\text { 1448610_a_at }\end{array}$ & & & & & & & & & I \\
\hline SOD3 & 1417633_at & & & & & & & & I & I \\
\hline SORBS2 & 1437197_at & & & & & & & I & I & I \\
\hline SOX 4 & $\begin{array}{l}\text { 1419155_a_at, } \\
\text { 1419156_at, } \\
\text { 1419157_at, } \\
\text { 1433575_at, } \\
\text { 1449370_at }\end{array}$ & & & & & $\mathrm{D}$ & $\mathrm{D}$ & $\mathrm{D}$ & $\mathrm{D}$ & $\mathrm{D}$ \\
\hline SP1 & $\begin{array}{l}\text { 1418180_at, } \\
\text { 1454852_at }\end{array}$ & & & & & & & & $\mathrm{D}$ & $\mathrm{D}$ \\
\hline SPP1 & 1449254_at & & & & & $\mathrm{D}$ & $\mathrm{D}$ & I & I & I \\
\hline SRGN & 1417426_at & & & & & & & & I & I \\
\hline STAT1 & $\begin{array}{l}\text { 1420915_at, } \\
\text { 1450033_a_at, } \\
\text { 1450034_at }\end{array}$ & & & & & & & $\mathrm{D}$ & $\mathrm{D}$ & $\mathrm{D}$ \\
\hline STAT5A & $\begin{array}{l}\text { 1421469_a_at, } \\
\text { 1450259_a_at }\end{array}$ & & & & & & & & I & I \\
\hline STAT6 & 1426353_at & & & & & & & & I & I \\
\hline STK24 & 1426248_at & & & & & & $\mathrm{D}$ & & & \\
\hline STMN1 & $\begin{array}{l}\text { 1415849_s_at, } \\
\text { 1448113_at }\end{array}$ & & & & & & & & $\mathrm{D}$ & $\mathrm{D}$ \\
\hline STX8 & 1418089_at & & & & & & & & I & I \\
\hline SULF1 & $\begin{array}{l}\text { 1436319_at, } \\
\text { 1438200_at }\end{array}$ & & & & & & & & & I \\
\hline TACC3 & $\begin{array}{l}\text { 1417450_a_at, } \\
\text { 1436872_at, } \\
\text { 1455834_x_at }\end{array}$ & & & & & & & & I & I \\
\hline TADA3L & 1417467_a_at & & & & & & & & & I \\
\hline TAX1BP1 & $\begin{array}{l}\text { 1420174_s_at, } \\
\text { 1448399_at }\end{array}$ & & & & & I & & & & I \\
\hline TCF12 & 1427670_a_at & & & & & & & & & $\mathrm{D}$ \\
\hline TCF4 & 1416724_x_at & & & & I & & & & & \\
\hline TCF7 & 1433471_at & & & & & & & & & I \\
\hline TENC1 & 1452264_at & & & $\mathrm{D}$ & & & & & & I \\
\hline
\end{tabular}


TABle 1: Continued.

\begin{tabular}{|c|c|c|c|c|c|c|c|c|c|c|}
\hline & & & $3 \mathrm{~h}$ & & & $24 \mathrm{~h}$ & & & $72 \mathrm{~h}$ & \\
\hline Symbol & Probe set ID & Gy & CDDP & $\begin{array}{l}\text { Gy }+ \\
\text { CDDP }\end{array}$ & Gy & CDDP & $\begin{array}{l}\text { Gy }+ \\
\text { CDDP }\end{array}$ & Gy & CDDP & $\begin{array}{l}\text { Gy }+ \\
\text { CDDP }\end{array}$ \\
\hline TERF1 & 1418380_at & & & & & & & & & I \\
\hline TFAP2A & $\begin{array}{l}\text { 1421996_at, } \\
\text { 1426048_s_at }\end{array}$ & & & & & & D & & & $\mathrm{D}$ \\
\hline TGFB1 & 1420653_at & & & & & & & & I & I \\
\hline TGFB1I1 & 1418136_at & & & & & & & & & I \\
\hline TGFB2 & 1450922_a_at & & & & & & & & I & I \\
\hline TGFBR2 & $\begin{array}{l}\text { 1425444_aaat, } \\
\text { 1426397_at }\end{array}$ & & & & & & & & I & I \\
\hline TGFBR3 & 1433795_at & & & & & $\mathrm{D}$ & & & & I \\
\hline THBS1 & $\begin{array}{l}\text { 1421811_at, } \\
\text { 1450377_at, } \\
\text { 1460302_at }\end{array}$ & & & $\mathrm{D}$ & & & & I & I & I \\
\hline THBS2 & $\begin{array}{l}\text { 1422571_at, } \\
\text { 1447862_x_at, } \\
\text { 1450663_at }\end{array}$ & & & D & & $\mathrm{D}$ & D & & & I \\
\hline TIAL1 & 1421148_a_at & & & & & & & $\mathrm{D}$ & & \\
\hline TIMP1 & 1460227_at & & & & & & & & I & I \\
\hline TIMP2 & $\begin{array}{l}\text { 1420924_at, } \\
\text { 1433662___at, } \\
\text { 1450040_at, } \\
\text { 1454677_at, } \\
\text { 1460287_at }\end{array}$ & & & & & & & I & I & I \\
\hline TIMP3 & $\begin{array}{l}\text { 1419088_at, } \\
\text { 1419089_at, } \\
\text { 1449334_at, } \\
\text { 1449335_at }\end{array}$ & & & & & D & & & I & I \\
\hline TLR1 & 1449049_at & & & & & & & & & I \\
\hline TLR3 & $\begin{array}{l}\text { 1422781_at, } \\
\text { 1422782_s_at }\end{array}$ & & & & & I & & D & I & I \\
\hline TLR4 & 1418163_at & & & & & & & & & I \\
\hline TMEM173 & $\begin{array}{l}\text { 1427911_at, } \\
\text { 1447621_s_at }\end{array}$ & & & & & & I & & I & I \\
\hline TMSB10 & $\begin{array}{l}\text { 1417219_s_at, } \\
\text { 1436902_x_at, } \\
\text { 1437185_s_at }\end{array}$ & & & & & & & I & & I \\
\hline TMSB4X & 1415906_at & & & & & & & I & I & I \\
\hline TNC & $\begin{array}{l}\text { 1416342_at, } \\
\text { 1456344_at }\end{array}$ & & & & & $\mathrm{D}$ & & & & I \\
\hline TNFAIP3 & 1433699_at & & & & & & & & I & I \\
\hline TNFAIP8 & 1416950_at & & & & & & & & I & I \\
\hline TNFRSF12A & $\begin{array}{l}\text { 1418571_at, } \\
\text { 1418572_x_at }\end{array}$ & & & & & & & I & I & I \\
\hline TNFRSF19 & 1425212_a_at & & & & & & & & I & \\
\hline TNFRSF1A & 1417291_at & & & D & & & & & & \\
\hline TNKS2 & 1447522_s_at & & & & & & & & I & I \\
\hline TOP1 & 1423474_at & & & & & I & & & & \\
\hline TOP2A & 1454694_a_at & & & & & I & I & & & \\
\hline TOPBP1 & 1452241_at & & & & & I & & & & I \\
\hline TOPORS & 1417754_at & & & & & & I & & & \\
\hline ТР53 & $\begin{array}{l}\text { 1426538_a_at, } \\
\text { 1427739_a_at }\end{array}$ & & & & & & & & & $\mathrm{D}$ \\
\hline
\end{tabular}


Table 1: Continued.

\begin{tabular}{|c|c|c|c|c|c|c|c|c|c|c|}
\hline & & & $3 \mathrm{~h}$ & & & $24 \mathrm{~h}$ & & & $72 \mathrm{~h}$ & \\
\hline Symbol & Probe set ID & Gy & CDDP & $\begin{array}{l}\text { Gy + } \\
\text { CDDP }\end{array}$ & Gy & CDDP & $\begin{array}{l}\text { Gy }+ \\
\text { CDDP }\end{array}$ & Gy & CDDP & $\begin{array}{l}\text { Gy + } \\
\text { CDDP }\end{array}$ \\
\hline ТР53BP2 & $\begin{array}{l}\text { 1433937_at, } \\
\text { 1433938_at }\end{array}$ & & & & & & & $\mathrm{D}$ & & $\mathrm{D}$ \\
\hline TP53INP1 & $\begin{array}{l}\text { 1416926_at, } \\
\text { 1416927_at }\end{array}$ & I & & I & & I & I & & I & I \\
\hline TPD52L1 & 1418412_at & & & & & & & & & I \\
\hline TPM1 & $\begin{array}{l}\text { 1423049_a_at, } \\
\text { 1423721_at }\end{array}$ & & & & & & & & I & I \\
\hline TPP1 & 1434768_at & & & & & & & & I & I \\
\hline TRAF3IP2 & 1448508_at & & & & & & & & & I \\
\hline TRAF7 & 1424320_a_at & & & & & & & & I & I \\
\hline TRIAP1 & 1460702_at & & & & & & & & & $\mathrm{D}$ \\
\hline TRIB2 & 1426640_s_at & & & & & $\mathrm{D}$ & $\mathrm{D}$ & & & \\
\hline TRIB3 & $\begin{array}{l}\text { 1426065_a_at, } \\
1456225 \_x \_a t\end{array}$ & & & & & & & $\mathrm{D}$ & $\mathrm{D}$ & $\mathrm{D}$ \\
\hline TRIM27 & $\begin{array}{l}\text { 1438376_s_at, } \\
\text { 1456375_x_at }\end{array}$ & & & & & & & & & $\mathrm{D}$ \\
\hline TSC2 & 1452105_a_at & & & & & & & & & I \\
\hline TSLP & 1450004_at & & & & & & & & I & I \\
\hline TSPO & $\begin{array}{l}\text { 1416695_at, } \\
\text { 1438948_x_at, } \\
\text { 1456251_x_at }\end{array}$ & & & & & & & & I & I \\
\hline TTK & 1449171_at & & & & & I & & & I & I \\
\hline TXN & 1416119_at & & & & & & & I & & \\
\hline TXNDC17 & $\begin{array}{l}\text { 1423034_at, } \\
\text { 1423035_s_at, } \\
\text { 1439184_s_at }\end{array}$ & & & & & & & & I & I \\
\hline TXNIP & $\begin{array}{l}\text { 1415996_at, } \\
\text { 1415997_at }\end{array}$ & & & & & I & & $\mathrm{D}$ & & $\mathrm{D}$ \\
\hline UBA7 & 1426971_at & & & & & & & & I & I \\
\hline UBE2C & 1452954_at & & & & & & & I & I & I \\
\hline UBR4 & 1454668_at & & & & & & & $\mathrm{D}$ & & \\
\hline UNG & 1425753_a_at & & & & & $\mathrm{D}$ & $\mathrm{D}$ & $\mathrm{D}$ & $\mathrm{D}$ & $\mathrm{D}$ \\
\hline UTP11L & 1429485_a_at & & & & & & & & & I \\
\hline UXT & 1418986_a_at & & & & & & & & & $\mathrm{D}$ \\
\hline VCAM1 & $\begin{array}{l}\text { 1415989_at, } \\
\text { 1436003_at, } \\
\text { 1448162_at, } \\
\text { 1451314_a_at }\end{array}$ & & & & & & & & I & I \\
\hline VCAN & 1427256_at & & & & & & & & & $\mathrm{D}$ \\
\hline VCL & $\begin{array}{l}\text { 1416156_at, } \\
\text { 1416157_at }\end{array}$ & & & & & & & & I & I \\
\hline VDR & $\begin{array}{l}\text { 1418175_at, } \\
\text { 1418176_at }\end{array}$ & & & & & & & & & I \\
\hline VHL & 1434708_at & & & & & & & $\mathrm{D}$ & & $\mathrm{D}$ \\
\hline WEE1 & 1416773_at & & & & & & & & & $\mathrm{D}$ \\
\hline WFS1 & 1448411_at & & & & & & & & & $\mathrm{D}$ \\
\hline
\end{tabular}


TABle 1: Continued.

\begin{tabular}{|c|c|c|c|c|c|c|c|c|c|c|}
\hline & & \multicolumn{3}{|c|}{$3 \mathrm{~h}$} & \multicolumn{3}{|c|}{$24 \mathrm{~h}$} & \multicolumn{3}{|c|}{$72 \mathrm{~h}$} \\
\hline Symbol & Probe set ID & Gy & CDDP & $\begin{array}{l}\text { Gy }+ \\
\text { CDDP }\end{array}$ & Gy & CDDP & $\begin{array}{l}\text { Gy }+ \\
\text { CDDP }\end{array}$ & Gy & CDDP & $\begin{array}{l}\text { Gy + } \\
\text { CDDP }\end{array}$ \\
\hline WISP1 & $\begin{array}{l}\text { 1448593_at, } \\
\text { 1448594_at }\end{array}$ & & & & & & & & I & I \\
\hline WRN & 1425982_a_at & & & & & & & & I & \\
\hline WTAP & 1454805_at & & & & & & & D & & $\mathrm{D}$ \\
\hline WWOX & 1416334_at & & & & & D & $\mathrm{D}$ & & & \\
\hline XAF1 & 1443698_at & & & & & & I & & & \\
\hline XBP1 & $\begin{array}{l}\text { 1420886_a_at, } \\
1437223 \text { _s_at }\end{array}$ & & & & & $\mathrm{D}$ & & & & \\
\hline $\mathrm{XDH}$ & 1451006_at & & & & & & & & I & \\
\hline XPA & 1460725_at & & & & & & & & & I \\
\hline XRCC2 & 1455335_at & & & & & & & & & D \\
\hline XRCC4 & 1424601_at & & & & & & & & D & D \\
\hline XRCC6 & 1417437_at & & & & & & & & $\mathrm{D}$ & D \\
\hline YARS & 1460638_at & & & & & & & & & D \\
\hline YWHAE & $\begin{array}{l}\text { 1435702_s_at, } \\
\text { 1438839_a_at }\end{array}$ & & & & & & & & & D \\
\hline YY1 & $\begin{array}{l}\text { 1435824_at, } \\
\text { 1457834_at }\end{array}$ & & & D & & & & & I & I \\
\hline ZFP36 & 1452519_a_at & & & D & & & & & I & \\
\hline ZFP36L2 & 1437626_at & & & & & D & $\mathrm{D}$ & & & I \\
\hline ZMAT3 & 1449353_at & & & I & & & & & I & I \\
\hline ZNF148 & $\begin{array}{l}\text { 1418381_at, } \\
\text { 1449068_at, } \\
\text { 1449069_at }\end{array}$ & & & & & I & I & & & \\
\hline ZNF622 & 1438000_x_at & & & & & & & & $\mathrm{D}$ & $\mathrm{D}$ \\
\hline ZYX & 1417240_at & & & & & & & I & I & I \\
\hline
\end{tabular}

after treatment, compared to CDDP-induced activation which was observed only at $24 \mathrm{hrs}$ or later (Figure 4). These timings corresponded with those observed for the expression of apoptotic-related genes after radiation and CDDP treatment (Figure 3). For example, MDM2 and TP53INP1 were expressed at $3 \mathrm{hrs}$ after radiation. They were however, expressed only at $24 \mathrm{hrs}$ after CDDP (Table 2).

\section{Discussion}

Combined chemoradiation is increasingly being used to treat advanced head and neck caners. As radiation and CDDP are both ototoxic, it is of concern that significant sensorineural hearing loss will result. Indeed, patients with nasopharyngeal carcinoma who had received radiotherapy and concurrent/adjuvant chemotherapy using CDDP were found to experience greater sensorineural hearing loss compared with patients treated with radiotherapy alone, especially to highfrequency sounds in the speech range [1]. It is of interest to note that different etiologies of sensorineural hearing loss, such as noise, ototoxic drugs, and aging, result in similar patterns of audiometric changes and cochlear cellular degeneration [8]. The cellular and molecular mechanisms involved in sensorineural hearing loss from diverse causes appear to lead to a final common pathway which results in apoptosis of cochlear hair cells $[6,9]$.

In radiation-induced ototoxicity, cochlear cell apoptosis and ROS generation were observed after irradiation, and p53 was thought to play a key role [7]. This phenomenon was dose dependant and occurred predominantly at $72 \mathrm{~h}$ after irradiation. Microarray analysis supported these findings, as associated dose-dependant apoptotic gene regulation changes were observed.

The ototoxic manifestations of CDDP are primarily due to its effects on the cochlear hair cells although the spiral ganglion cells and the stria vascularis are also affected to some extent. According to Rybak et al. [10], CDDP ototoxicity appears to be triggered by ROSs that initiate a cascade of molecular events that lead to apoptosis of outer hair cells, resulting in hearing loss. Ototoxic effects on the stria vascularis are transient, resulting in temporary reduction of endocochlear potential associated with stria edema. The endocochlear potential recovers but residual shrinkage of the strial persists. The spiral ganglia are thought to be least affected.

Although the cellular and molecular processes of ototoxicity have been described for radiation and CDDP when used alone, those involved in combined therapy have not been 
TABLE 2: Differential expression of apoptosis-related genes which have direct upstream or downstream relationship with p53 in each treatment group [irradiation (Gy), cisplatin (CDDP), or combination of both (Gy + CDDP)] when compared to nontreated control cells at $3 \mathrm{~h}, 24 \mathrm{~h}$, and $72 \mathrm{~h}$ after treatment.

\begin{tabular}{|c|c|c|c|c|c|c|c|}
\hline \multicolumn{8}{|c|}{3 hours } \\
\hline Symbol & Gy & CDDP & $\mathrm{Gy}+\mathrm{CDDP}$ & Symbol & Gy & CDDP & $\mathrm{Gy}+\mathrm{CDDP}$ \\
\hline CCNG1 & I & & I & KLF6 & $\mathrm{D}$ & $\mathrm{D}$ & $\mathrm{D}$ \\
\hline CDKN1A & I & & I & CCND1 & $\mathrm{D}$ & & \\
\hline MDM2 & I & & I & CTCF & $\mathrm{D}$ & & \\
\hline TP53INP1 & I & & I & IRS1 & $\mathrm{D}$ & & \\
\hline BTG2 & I & & & ATF3 & & & $\mathrm{D}$ \\
\hline DDX5 & I & & & AURKA & & & $\mathrm{D}$ \\
\hline GDF15 & I & & & BID & & & $\mathrm{D}$ \\
\hline IL6 & I & & & CCNB1 & & & $\mathrm{D}$ \\
\hline C11ORF82 & & & I & СЕВРВ & & & $\mathrm{D}$ \\
\hline CASP3 & & & I & DDR1 & & & $\mathrm{D}$ \\
\hline CASP6 & & & I & ETS1 & & & $\mathrm{D}$ \\
\hline CRYAB & & & I & FHL2 & & & $\mathrm{D}$ \\
\hline HSP90AA1 & & & I & HBEGF & & & $\mathrm{D}$ \\
\hline MED1 & & & I & HIPK1 & & & $\mathrm{D}$ \\
\hline \multirow[t]{12}{*}{ ZMAT3 } & & & I & HSPB1 & & & $\mathrm{D}$ \\
\hline & & & & MAPK3 & & & $\mathrm{D}$ \\
\hline & & & & MCL1 & & & $\mathrm{D}$ \\
\hline & & & & MYC & & & $\mathrm{D}$ \\
\hline & & & & NR4A1 & & & $\mathrm{D}$ \\
\hline & & & & OSGIN1 & & & $\mathrm{D}$ \\
\hline & & & & PLK1 & & & $\mathrm{D}$ \\
\hline & & & & PMEPA1 & & & $\mathrm{D}$ \\
\hline & & & & PPP1R13L & & & $\mathrm{D}$ \\
\hline & & & & THBS1 & & & $\mathrm{D}$ \\
\hline & & & & THBS2 & & & $\mathrm{D}$ \\
\hline & & & & YY1 & & & $\mathrm{D}$ \\
\hline \multicolumn{8}{|c|}{24 hours } \\
\hline Symbol & Gy & CDDP & Gy+CDDP & Symbol & Gy & CDDP & $\mathrm{Gy}+\mathrm{CDDP}$ \\
\hline CCNG1 & I & I & I & APEX1 & & $\mathrm{D}$ & D \\
\hline CDKN1A & I & I & I & BHLHE40 & & $\mathrm{D}$ & $\mathrm{D}$ \\
\hline EIF2AK2 & I & I & I & BRE & & $\mathrm{D}$ & $\mathrm{D}$ \\
\hline $\mathrm{RFC1}$ & I & I & I & PMEPA1 & & $\mathrm{D}$ & $\mathrm{D}$ \\
\hline BUB1 & & I & I & S100A4 & & $\mathrm{D}$ & $\mathrm{D}$ \\
\hline CCAR1 & & I & I & SERPINE1 & & $\mathrm{D}$ & $\mathrm{D}$ \\
\hline CKAP2 & & I & I & SPP1 & & $\mathrm{D}$ & $\mathrm{D}$ \\
\hline HSP90AA1 & & I & I & THBS2 & & $\mathrm{D}$ & $\mathrm{D}$ \\
\hline MDM2 & & I & I & WwOX & & $\mathrm{D}$ & $\mathrm{D}$ \\
\hline TOP2A & & I & I & SLC2A1 & $\mathrm{D}$ & & \\
\hline TP53INP1 & & I & I & BTG1 & & $\mathrm{D}$ & \\
\hline ZNF148 & & I & I & CAV1 & & $\mathrm{D}$ & \\
\hline KLF6 & I & & & СЕВРВ & & $\mathrm{D}$ & \\
\hline BLM & & I & & TIMP3 & & $\mathrm{D}$ & \\
\hline
\end{tabular}


TABle 2: Continued.

\begin{tabular}{|c|c|c|c|c|c|c|c|}
\hline \multicolumn{8}{|c|}{24 hours } \\
\hline Symbol & Gy & CDDP & Gy+CDDP & Symbol & Gy & CDDP & $\mathrm{Gy}+\mathrm{CDDP}$ \\
\hline BRCA1 & & I & & ATP1A1 & & & $\mathrm{D}$ \\
\hline HMMR & & I & & CDK4 & & & $\mathrm{D}$ \\
\hline IFI16 & & I & & CDKN2A & & & $\mathrm{D}$ \\
\hline RAD54L & & I & & CDKN2C & & & $\mathrm{D}$ \\
\hline RB1 & & I & & GSTM5 & & & $\mathrm{D}$ \\
\hline TOP1 & & I & & ID2 & & & $\mathrm{D}$ \\
\hline TOPBP1 & & I & & TFAP2A & & & $\mathrm{D}$ \\
\hline TTK & & I & & & & & \\
\hline BTG2 & & & I & & & & \\
\hline C11ORF82 & & & I & & & & \\
\hline FUBP1 & & & I & & & & \\
\hline NFKB2 & & & I & & & & \\
\hline NUPR1 & & & I & & & & \\
\hline TOPORS & & & I & & & & \\
\hline \multicolumn{8}{|c|}{72 hours } \\
\hline Symbol & Gy & CDDP & Gy+CDDP & Symbol & Gy & CDDP & Gy+CDDP \\
\hline ANXA1 & I & I & I & LGALS3 & & & I \\
\hline AURKA & I & I & I & LIF & & & I \\
\hline BUB1B & I & I & I & MAP2K3 & & & I \\
\hline CAV1 & I & I & I & MMP2 & & & I \\
\hline CCNB1 & I & I & I & MYO6 & & & I \\
\hline CCND1 & I & I & I & NFKB1 & & & I \\
\hline CCNG1 & I & I & I & PLAUR & & & I \\
\hline CDC20 & I & I & I & PRKCA & & & I \\
\hline CDKN1A & I & I & I & PTGS1 & & & I \\
\hline CLU & I & I & I & SAT1 & & & I \\
\hline DDIT4 & I & I & I & SERPINE2 & & & I \\
\hline EGR1 & I & I & I & SLC2A1 & & & I \\
\hline FOSL1 & I & I & I & SOD2 & & & I \\
\hline GLIPR1 & I & I & I & TADA3L & & & I \\
\hline HSPB1 & I & I & I & THBS2 & & & I \\
\hline IER3 & I & I & I & TOPBP1 & & & I \\
\hline INHBA & I & I & I & TSC2 & & & I \\
\hline IRS1 & I & I & I & VDR & & & I \\
\hline NFKBIA & I & I & I & FEN1 & $\mathrm{D}$ & $\mathrm{D}$ & $\mathrm{D}$ \\
\hline PHLDA1 & I & I & I & GADD45A & $\mathrm{D}$ & $\mathrm{D}$ & $\mathrm{D}$ \\
\hline PLK1 & I & I & I & JUN & $\mathrm{D}$ & D & $\mathrm{D}$ \\
\hline PTGS2 & I & I & I & MCM2 & $\mathrm{D}$ & $\mathrm{D}$ & $\mathrm{D}$ \\
\hline PTTG1 & I & I & I & NDRG1 & D & $\mathrm{D}$ & $\mathrm{D}$ \\
\hline S100A4 & I & I & I & STAT1 & D & $\mathrm{D}$ & $\mathrm{D}$ \\
\hline SERPINE1 & I & I & I & ATF3 & $\mathrm{D}$ & & $\mathrm{D}$ \\
\hline SGK1 & I & I & I & PPP2R2A & $\mathrm{D}$ & & $\mathrm{D}$ \\
\hline SPP1 & I & I & I & ТР53ВР2 & $\mathrm{D}$ & & $\mathrm{D}$ \\
\hline THBS1 & I & I & I & CDK4 & & D & $\mathrm{D}$ \\
\hline TMSB4X & I & I & I & FUBP1 & & $\mathrm{D}$ & $\mathrm{D}$ \\
\hline UBE2C & I & I & I & GSK3B & & D & $\mathrm{D}$ \\
\hline ZYX & I & I & I & HMGB1L1 & & $\mathrm{D}$ & $\mathrm{D}$ \\
\hline BCL3 & I & & I & HSPA5 & & $\mathrm{D}$ & $\mathrm{D}$ \\
\hline MMP3 & I & & I & PARK7 & & $\mathrm{D}$ & $\mathrm{D}$ \\
\hline S100A6 & I & & I & PCNA & & $\mathrm{D}$ & $\mathrm{D}$ \\
\hline
\end{tabular}


Table 2: Continued.

\begin{tabular}{|c|c|c|c|c|c|c|c|}
\hline \multicolumn{8}{|c|}{72 hours } \\
\hline Symbol & Gy & CDDP & Gy+CDDP & Symbol & Gy & CDDP & Gy+CDDP \\
\hline ABCB1B & & I & I & PPP1R15A & & $\mathrm{D}$ & $\mathrm{D}$ \\
\hline AKAP12 & & I & I & SMN1 & & D & $\mathrm{D}$ \\
\hline ATM & & I & I & SP1 & & $\mathrm{D}$ & D \\
\hline BID & & I & I & STMN1 & & $\mathrm{D}$ & $\mathrm{D}$ \\
\hline BIRC5 & & I & I & XRCC6 & & $\mathrm{D}$ & $\mathrm{D}$ \\
\hline BUB1 & & I & I & DAXX & $\mathrm{D}$ & I & \\
\hline CCNA2 & & I & I & MX1 & $\mathrm{D}$ & I & \\
\hline CCND3 & & I & I & PML & $\mathrm{D}$ & I & \\
\hline $\mathrm{CDC} 25 \mathrm{C}$ & & I & I & $\mathrm{E} 2 \mathrm{~F} 1$ & $\mathrm{D}$ & & \\
\hline CKAP2 & & I & I & IFI16 & $\mathrm{D}$ & & \\
\hline CRYAB & & I & I & BRE & & & $\mathrm{D}$ \\
\hline CTSD & & I & I & CDC6 & & & $\mathrm{D}$ \\
\hline DDR1 & & I & I & CHEK1 & & & $\mathrm{D}$ \\
\hline DHCR24 & & I & I & COPS5 & & & $\mathrm{D}$ \\
\hline EZR & & I & I & CTCF & & & $\mathrm{D}$ \\
\hline FHL2 & & I & I & DDIT3 & & & $\mathrm{D}$ \\
\hline FOS & & I & I & DUT & & & $\mathrm{D}$ \\
\hline FOXM1 & & I & I & ELAVL1 & & & $\mathrm{D}$ \\
\hline HMMR & & I & I & HOXA7 & & & $\mathrm{D}$ \\
\hline IL6 & & I & I & HUWE1 & & & $\mathrm{D}$ \\
\hline KAT2B & & I & I & KAT5 & & & $\mathrm{D}$ \\
\hline KLF4 & & I & I & MAPK8 & & & $\mathrm{D}$ \\
\hline MDM2 & & I & I & NME1 & & & $\mathrm{D}$ \\
\hline MET & & I & I & RBBP6 & & & $\mathrm{D}$ \\
\hline NEK2 & & I & I & TFAP2A & & & $\mathrm{D}$ \\
\hline NQO1 & & I & I & TP53 & & & $\mathrm{D}$ \\
\hline NQO2 & & I & I & VCAN & & & $\mathrm{D}$ \\
\hline NR3C1 & & I & I & & & & \\
\hline PLK3 & & I & I & & & & \\
\hline PTPRA & & I & I & & & & \\
\hline RAD54L & & I & I & & & & \\
\hline S100A1 & & I & I & & & & \\
\hline SHISA5 & & I & I & & & & \\
\hline ТАСС3 & & I & I & & & & \\
\hline TGFB2 & & I & I & & & & \\
\hline TIMP3 & & I & I & & & & \\
\hline TP53INP1 & & I & I & & & & \\
\hline TTK & & I & I & & & & \\
\hline YY1 & & I & I & & & & \\
\hline ZMAT3 & & I & I & & & & \\
\hline TXN & I & & & & & & \\
\hline GDF15 & & I & & & & & \\
\hline GSTM1 & & I & & & & & \\
\hline RFWD2 & & I & & & & & \\
\hline RRM2B & & I & & & & & \\
\hline WRN & & I & & & & & \\
\hline AFP & & & I & & & & \\
\hline AHR & & & I & & & & \\
\hline AP2A2 & & & I & & & & \\
\hline BHLHE40 & & & I & & & & \\
\hline C11ORF82 & & & I & & & & \\
\hline
\end{tabular}


TABle 2: Continued.

\begin{tabular}{|c|c|c|c|c|c|c|c|}
\hline \multicolumn{8}{|c|}{72 hours } \\
\hline Symbol & Gy & CDDP & Gy+CDDP & Symbol & Gy & CDDP & $\mathrm{Gy}+\mathrm{CDDP}$ \\
\hline CASP6 & & & I & & & & \\
\hline CAT & & & I & & & & \\
\hline CDK8 & & & I & & & & \\
\hline CENPF & & & I & & & & \\
\hline CFLAR & & & I & & & & \\
\hline CSF2 & & & I & & & & \\
\hline CX3CL1 & & & I & & & & \\
\hline EPHA2 & & & I & & & & \\
\hline ERCC3 & & & I & & & & \\
\hline ERCC5 & & & I & & & & \\
\hline ETS1 & & & I & & & & \\
\hline FAS & & & I & & & & \\
\hline FASN & & & I & & & & \\
\hline GPI & & & I & & & & \\
\hline HK2 & & & I & & & & \\
\hline HSP90AB1 & & & I & & & & \\
\hline ID1 & & & I & & & & \\
\hline
\end{tabular}

Differentially expressed apoptotic related genes
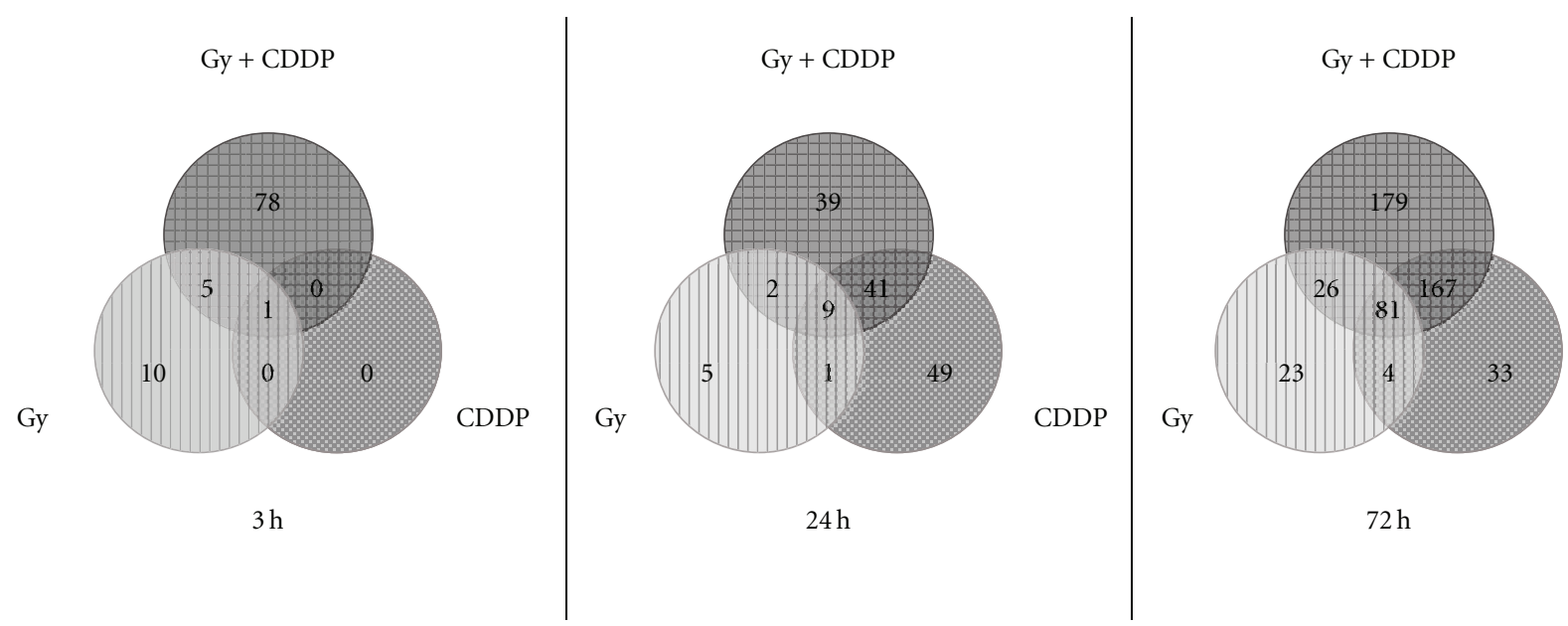

CDDP

FIGURE 3: Microarray findings are summarized by Venn diagrams which show the distribution of differentially expressed probeset IDs in each treatment group [irradiation (Gy), cisplatin (CDDP) or combination of both (Gy and CDDP)] when compared to nontreated control cells at $3 \mathrm{~h}, 24 \mathrm{~h}$, and $72 \mathrm{~h}$ after treatment.

studied previously. The present study demonstrated that combined therapy led to decreased viability of cochlear cells, with an increase in the subG1 population. These findings support the belief that as in other etiologies of sensorineural loss, apoptosis of cochlear hair cells is important in CDDPradiation.

It is well established that p53 plays a key role in the cellular response to nuclear DNA damage [11]. It regulates cell cycle arrest and dictates cell fate like senescence, apoptosis, and DNA repair. It is believed that the nature of DNA damage enables p53 to selectively discriminate between promotors in the induction of target genes, thereby regulating their expression and subsequent cellular outcome [12].

In a study on HEI-OC1 cells derived from the cochlea, CDDP caused an increase in p53 at $3 \mathrm{hrs}$ prior to the activation of Bax, cytochrome-c, and caspase 8 and 9 [13]. In the case of radiation-induced ototoxicity, the role of p53 in triggering apoptotic cell death in cochlear hair cells has also been studied [7]. Based on microarray analysis, the p53 gene was found to be up-regulated after irradiation and p53 


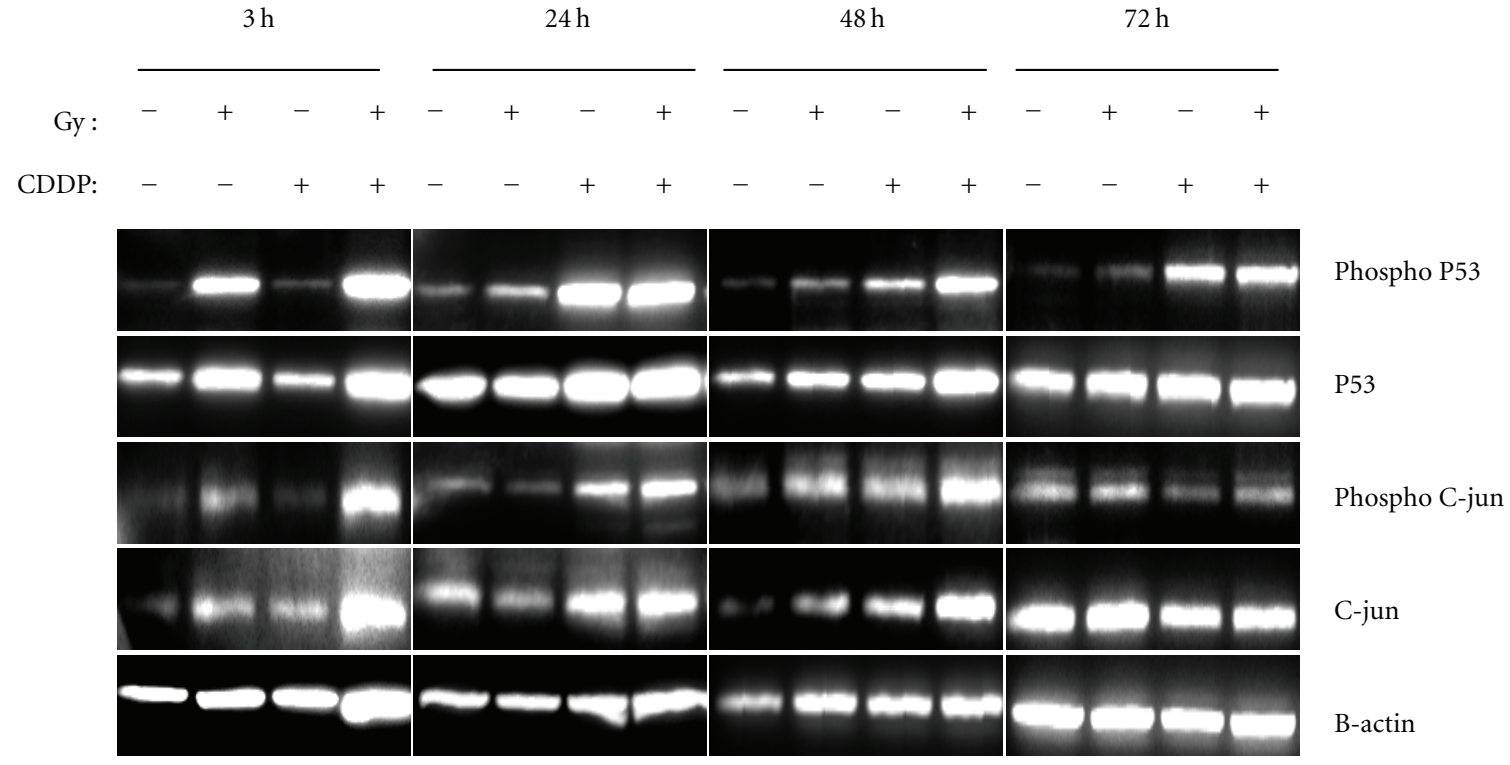

Figure 4: Western blot analysis showing p53 and c-jun protein expression and phosphorylation at various time points ( $3 \mathrm{~h}, 24 \mathrm{~h}, 48 \mathrm{~h}$, and $72 \mathrm{~h}$ ) after $5 \mathrm{~Gy}$ of gamma radiation and $0.5 \mu \mathrm{g} / \mathrm{ml}$ pf cisplatin (CDDP). The data are representative of 3 separate experiments.

expression was confirmed by Western blotting. Although p53 plays a role in both CDDP and radiation-induced ototoxicity, the present study showed that p53 was activated at different time points after treatment. Posttreatment phosphorylation of p53 occurred after 24 hrs for CDDP, whereas it occurred as early as $3 \mathrm{hrs}$ for radiation. These timings corresponded to the times MDM2 and TP53INP1 were expressed after treatment with CDDP and radiation respectively. Therefore, although both CDDP and radiation-induced cochlear cell apoptosis appear to involve activation of p53, the upstream processes involved may well be different.

In the present study, combined CDDP-radiation treatment triggered more apoptotic-related gene expressions than those that could be accounted for by a summation of gene expressions resulting from individual treatments. This could explain the synergistic ototoxic effects of combined CDDP-radiation treatment, an observation seen clinically [1]. Interestingly, among the genes which were expressed in combined treatment but not when these entities were used alone was FAS, a key element involved in the extrinsic apoptotic pathway. Although the extrinsic apoptotic pathway has generally been regarded to play only minor role in ototoxicity resulting from the use of CDDP or radiation alone, it may well be important in situations when they are used in combination $[14,15]$.

The OC-k3 cell line expressed the neuroepithelial precursor cell marker nestin and the inner ear cell marker OCP2, specific auditory sensory cell markers myosin VIIa and the acetylcholine receptor alpha- 9 and the supporting cell marker connexin 26. It had been regarded as a good model to study the mechanisms of cell fate in the Organ of Corti of the cochlea [4]. Therefore, the finding that combined treatment actually led to enhanced apoptotic gene expressions including FAS should be further investigated in in vivo animal studies which may have implications in future antiapoptotic treatments against ototoxicity.

\section{Conclusion}

Like in other etiologies of sensorineural loss, apoptosis of cochlear hair cells appears to play a role in ototoxicity resulting from combined CDDP-radiation therapy. Differential temporal activation of p53 suggests the possibility of different upstream processes leading to its activation after CDDP and radiation treatment. Enhanced apoptotic gene expressions including that of FAS were observed in combined treatment which could possibly explain the synergistic ototoxic effects seen clinically.

\section{Acknowledgments}

This study has been supported by a grant from the Department of Clinical Research, Singapore General Hospital. The authors thank Dr F. Kalinec (House Ear Institute, LA, USA) for providing the cell line and Mr Alvin WC Chua (Department of Plastic Reconstructive \& Aesthetic Surgery, Singapore General Hospital) for his guidance in cell culture.

\section{References}

[1] W. K. Low, S. T. Toh, J. Wee, S. M. C. Fook-Chong, and D. Y. Wang, "Sensorineural hearing loss after radiotherapy and chemoradiotherapy: a single, blinded, randomized study," Journal of Clinical Oncology, vol. 24, no. 12, pp. 1904-1909, 2006.

[2] M. N. Rivolta and M. C. Holley, "Cell lines in inner ear research,” Journal of Neurobiology, vol. 53, no. 2, pp. 306-318, 2002. 
[3] F. Kalinec, G. Kalinec, M. Boukhvalova, and B. Kachar, "Establishment and characterization of conditionally immortalized organ of Corti cell lines," Cell Biology International, vol. 23, no. 3, pp. 175-184, 1999.

[4] G. M. Kalinec, P. Webster, D. J. Lim, and F. Kalinec, "A cochlear cell line as an in vitro system for drug ototoxicity screening," Audiology and Neuro-Otology, vol. 8, no. 4, pp. 177-189, 2003.

[5] M. Zhang, W. Liu, D. Ding, and R. Salvi, "Pifithrin- $\alpha$ supresses p53 and protects cochlear and vestibular hair cells from cisplatin-induced apoptosis," Neuroscience, vol. 120, no. 1, pp. 191-205, 2003.

[6] A. G. Cheng, L. L. Cunningham, and E. W. Rubel, "Mechanisms of hair cell death and protection," Current Opinion in Otolaryngology and Head and Neck Surgery, vol. 13, no. 6, pp. 343-348, 2005.

[7] W.-K. Low, M. G. K. Tan, L. Sun, A. W. C. Chua, L.-K. Goh, and D.-Y. Wang, "Dose-dependant radiation-induced apoptosis in a cochlear cell-line," Apoptosis, vol. 11, no. 12, pp. 2127-2136, 2006.

[8] D. Henderson, E. C. Bielefeld, K. C. Harris, and B. H. Hu, "The role of oxidative stress in noise-induced hearing loss," Ear and Hearing, vol. 27, no. 1, pp. 1-19, 2006.

[9] O. Atar and K. B. Avraham, "Therapeutics of hearing loss: expectations vs reality," Drug Discovery Today, vol. 10, no. 19, pp. 1323-1330, 2005.

[10] L. P. Rybak, C. A. Whitworth, D. Mukherjea, and V. Ramkumar, "Mechanisms of cisplatin-induced ototoxicity and prevention," Hearing Research, vol. 226, no. 1-2, pp. 157$167,2007$.

[11] R. G. Bristow, S. Benchimol, and R. P. Hill, "The p53 gene as a modifier of intrinsic radiosensitivity: implications for radiotherapy," Radiotherapy and Oncology, vol. 40, no. 3, pp. 197-223, 1996.

[12] R. Hill, E. Bodzak, M. D. Blough, and P. W. K. Lee, "p53 binding to the $\mathrm{p} 21$ promoter is dependent on the nature of DNA damage," Cell Cycle, vol. 7, no. 16, pp. 2535-2543, 2008.

[13] P. Devarajan, M. Savoca, M. P. Castaneda et al., "Cisplatininduced apoptosis in auditory cells: role of death receptor and mitochondrial pathways," Hearing Research, vol. 174, no. 1-2, pp. 45-54, 2002.

[14] J. Wang, S. Ladrech, R. Pujol, P. Brabet, T. R. Van De Water, and J.-L. Puel, "Caspase inhibitors, but not c-Jun $\mathrm{NH}_{2}$ terminal kinase inhibitor treatment, prevent cisplatin-induced hearing loss," Cancer Research, vol. 64, no. 24, pp. 9217-9224, 2004.

[15] M. Verheij and H. Bartelink, "Radiation-induced apoptosis," Cell and Tissue Research, vol. 301, no. 1, pp. 133-142, 2000. 


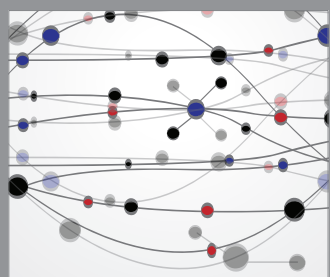

The Scientific World Journal
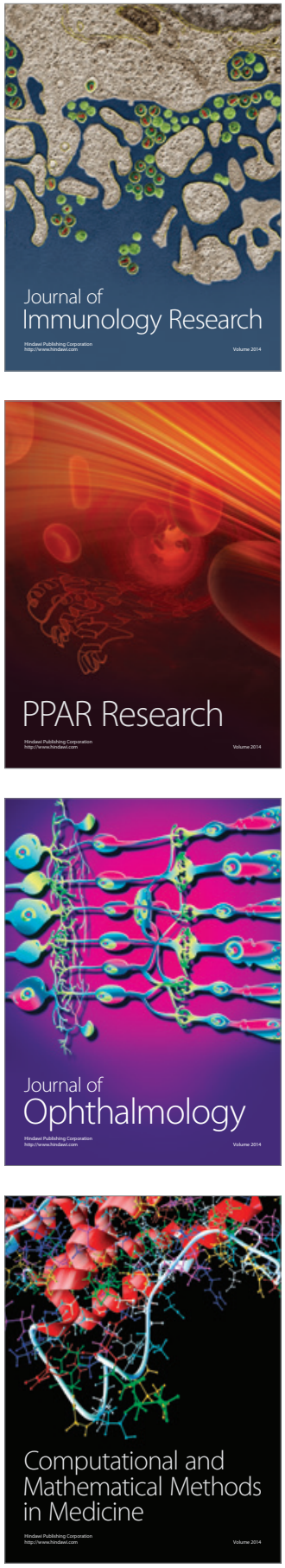

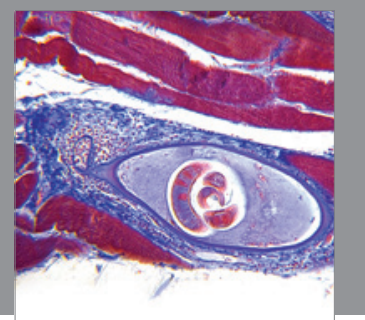

Gastroenterology

Research and Practice
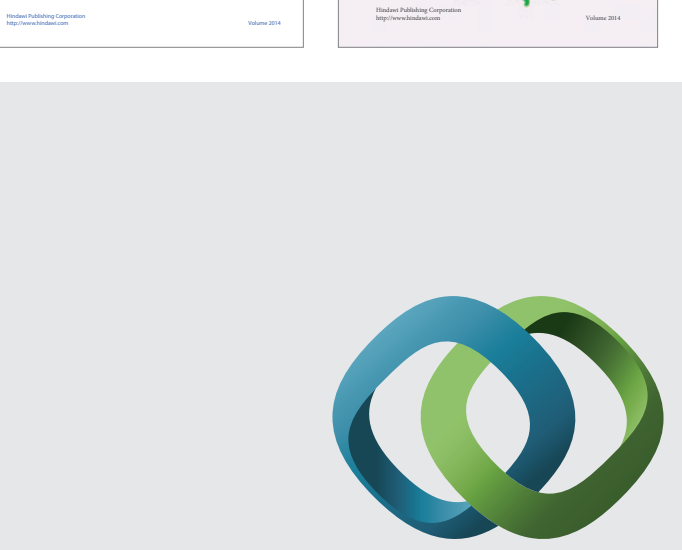

\section{Hindawi}

Submit your manuscripts at

http://www.hindawi.com
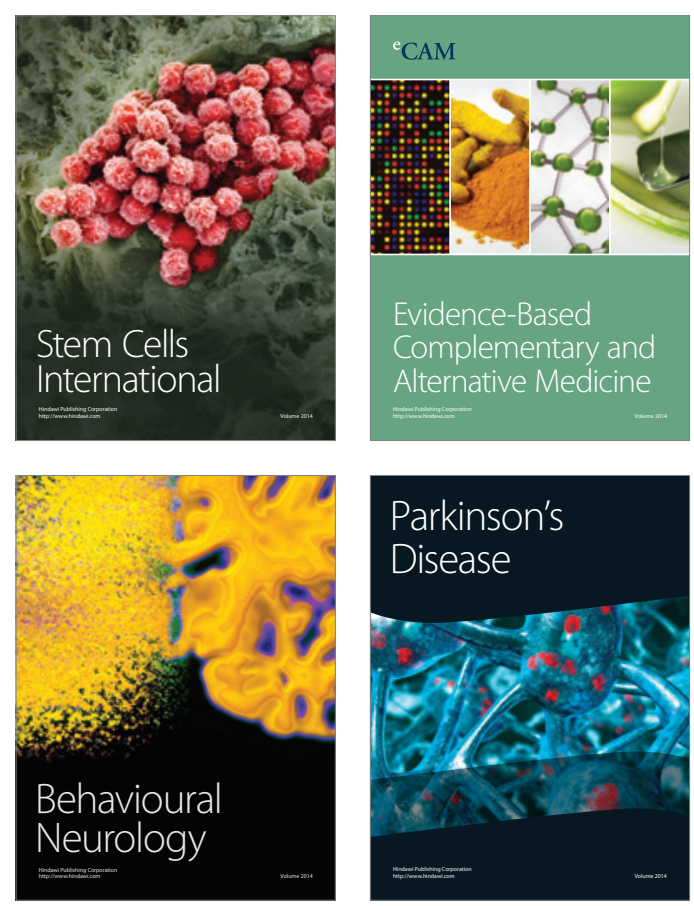

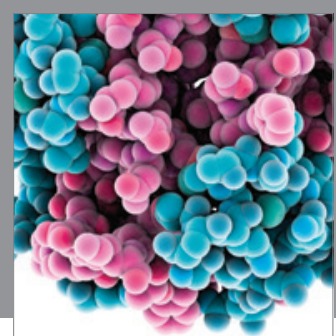

Journal of
Diabetes Research

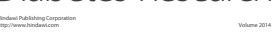

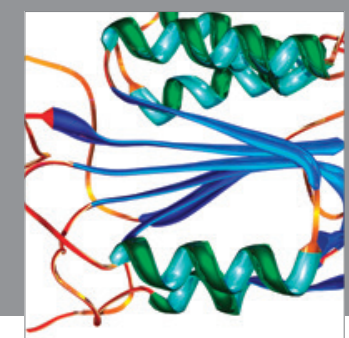

Disease Markers
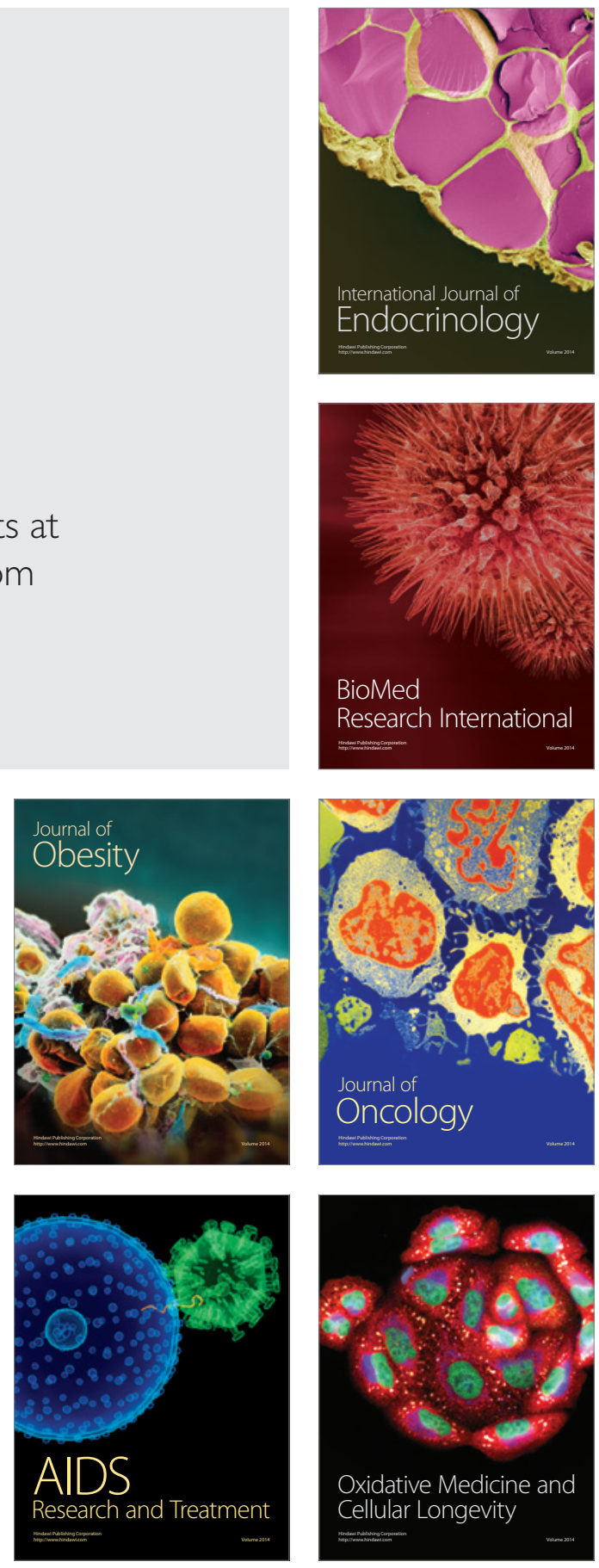